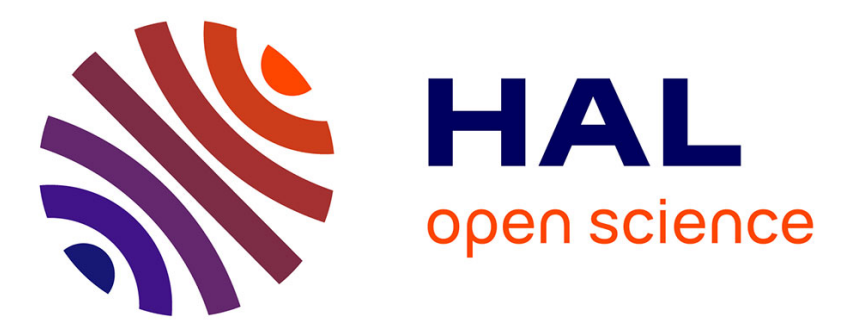

\title{
Boundary conditions for the computation of thermo-acoustic modes in combustion chambers
}

Camilo Silva, Ignacio Duran, Franck Nicoud, Stéphane Moreau

\section{To cite this version:}

Camilo Silva, Ignacio Duran, Franck Nicoud, Stéphane Moreau. Boundary conditions for the computation of thermo-acoustic modes in combustion chambers. AIAA Journal, 2014, 52 (6), pp.1180-1193. 10.2514/1.J052114 . hal-01056693

\section{HAL Id: hal-01056693 https://hal.science/hal-01056693}

Submitted on 20 Aug 2014

HAL is a multi-disciplinary open access archive for the deposit and dissemination of scientific research documents, whether they are published or not. The documents may come from teaching and research institutions in France or abroad, or from public or private research centers.
L'archive ouverte pluridisciplinaire HAL, est destinée au dépôt et à la diffusion de documents scientifiques de niveau recherche, publiés ou non, émanant des établissements d'enseignement et de recherche français ou étrangers, des laboratoires publics ou privés. 


\title{
Boundary conditions for the computation of
}

\section{thermo-acoustic modes in combustion chambers}

\author{
Camilo F. Silva ${ }^{1}$ \\ Technische Universität München D-85747 Garching \\ Ignacio Durán ${ }^{2}$ \\ CERFACS, 31057 Toulouse, France \\ Franck Nicoud ${ }^{3}$ \\ Université Montpellier II, 34095 Montpellier, France
}

Stéphane Moreau 4

Université Sherbrooke, 2500, boul. de l'Université Sherbrooke (Québec), Canada J1K 2R1

In order to carry out reliable LES or Helmholtz solver computations in aeronautical combustion chambers, it is crucial to impose the right boundary conditions at both inlet and outlet of the combustion system. This means providing acoustic boundary impedances that take into account all the stages in compressors and turbines. This study is a follow up of works where the boundary condition were derived from the isentropic and isenthalpic Linearized Euler Equations. Specific terms are added to the quasi-1D LEE in order to account for enthalpy jumps induced by the rotor stages of the compressors and turbines. This system of equations also accounts for the entropyacoustic coupling since, in addition to the continuity and momentum equations, the entropy equation is also considered. The numerical implementation is validated by comparing the numerical results with analytical solutions in several model problems. The effective impedance of the inlet air-circuit of an actual helicopter engine is then

\footnotetext{
1 Corresponding author. Post-doctoral fellow, Technische Universität München, kamilosilva@gmail.com. Tel: $+49(0) 89.289 .16239$

2 Ph.D. Student, Cerfacs CFD Team, 42, Av. Gaspard Coriolis; duran@cerfacs.fr. Tel: +33.(0)5.61.19.30.22

3 Professor, Université Montpellier II, I3M - CNRS UMR 5149 - CC51, franck.nicoud@univ-montp2.fr

4 Professor, GAUS, Université de Sherbrooke, stephane.moreau@usherbrooke.ca.
} 
computed in order to illustrate the potential of the method as well as the importance of the rotor terms added.

\section{Introduction}

The prediction of combustion instabilities in combustion chambers embedded in complex systems, such as aeronautical engines, is extremely difficult because different phenomena must be taken into account at once: the strong interaction between the flame and the most representative turbulent length scales of the reacting and burnt gases, the two-way interaction between the flame and the radiated acoustic waves it produces, the possible coupling between entropy waves caused by the hot spots generated by the flame and the acoustic waves produced by the non-homogeneities in the mean flow, and evidently the role played by all boundary conditions (inlets, outlets and walls).

Different strategies have been developed over the years [1-5] to understand the physics of all these interactions and to create methodologies to control or avoid them. In recent years, reacting and compressible Large Eddy-Simulations (LES) have shown their capability to study the dynamics of turbulent flames [6-12]. Due to its intrinsic nature (resolution of the unsteady 3D Navier-Stokes equations) LES is able to predict the interactions between the flame, the turbulent flow and the acoustic modes of the chamber, and for some particular configurations to distinguish between stable and unstable combustion systems [13]. Yet, LES presently remains very CPU demanding and its use for parametric studies of aeronautical engines design is still excluded.

For this reason, simplifications on the geometry of the combustor as well as on the physics of the reactive turbulent flow may lead to interesting and useful strategies to study combustion instabilities. Following this philosophy, a wide spread methodology has been developed over the years which is based on the so called low-order models. A given system is characterized as a network of individual one dimensional/ two dimensional axisymmetric acoustic elements in which only perturbations of first order are kept [14-17]. Since acoustics is considered to be the only cause of these perturbations, all viscous terms in the Navier Stokes equations are neglected. The acoustic elements are then described by 1D Linearized Euler Equations (LEE) and they are associated with 
each other by relations of conservation of mass, momentum and energy. These methods aim to study the low frequency unstable modes that are originated by the coupling of plane acoustic waves with compact heat release sources. For complex geometries, as actual aeronautical combustion chambers, this 1D modeling strategy might remain too simplistic: the full three dimensional geometry might play an important role not only on the values of the predicted resonance frequencies but also on the growth rate of the acoustic modes, as shown for example in [18]). Instead of solving the LEE equations for a set of interconnected acoustic elements, it might be more precise to account for the actual 3D geometry. The development of LEE solvers for complex geometries associated to combustion chambers is still in a nascent state. In such an approach the stability not only of acoustic modes, but also vortical and entropy modes is studied [19]. Nevertheless, the mean flow velocity field in typical combustion chambers is very low, and assuming that convective terms do not significantly contribute in the analysis of combustion instabilities is reasonable. Therefore, under the low Mach approximation, the LEE equations can be recast into a wave equation that if expressed in the frequency domain results in the so called Helmhotz Equation. The respective numerical tools, commonly known as Helmholtz solvers, have proved their ability to predict combustion instabilities $[13,20,21]$ in real combustion chambers.

The ability of the strategies above described (LES, low-order model techniques and Helmholtz solvers) to correctly estimate combustion instabilities depends strongly on the acoustic description at the system boundaries. The main objective when describing the acoustic behaviour of such boundaries is to be able to predict accurately the transmission and reflection of acoustic waves caused by impinging acoustic waves at these frontiers. The general idea is to solve the quasi-1D LEE in a domain that represents the regions either upstream (fan + compressor stages) or downstream (turbine stages + nozzle) of the combustion chamber. Several works have made important contributions in the study of acoustics of quasi 1D elements. The first significant study is due to Tsien [22]. In this work, the propagation of acoustic waves through a quasi 1D system is studied. The mean flow is considered to be linearly distributed along the axis. Subsequently, Marble and Candel [23] proposed analytical expressions that estimate the transmission and reflection of acoustic waves due to either acoustic or entropy waves that are injected in a quasi $1 \mathrm{D}$ system. The main assumption, in addition 
to consider plane acoustic waves, is to consider the system as compact, i.e. as a system whose length is considered small compared with a characteristic acoustic wavelength. These relations have been demonstrated to be very useful in the low frequency limit. In the work of Stow et al. [24], this theory is extended to second-order through an asymptotic analysis. A second-order correction is added through an effective length which is function of the mean flow spatial distribution. Consequently, the transmission and reflection coefficients are well predicted within a low frequency band. In the study of Moase et al. [25], transmission and reflection coefficients are derived for a quasi-1D system whose mean flow is modelled to be piece-wise linear. As a result, an acoustic characterization of any quasi 1D geometry can be performed for any frequency band of interest as long as the mean flow can be described by piece-wise linear functions. Recently, a remarkable work has been carried out by Duran and Moreau [26] in which the quasi 1D LEE are solved analytically through an asymptotic analysis based on the Magnus expansion of the quasi 1D LEE. In addition to analytical methods, numerical approaches to solve the quasi 1D LEE system can be also used to study the acoustic properties of a given quasi 1D system. Corresponding numerical schemes have demonstrated to be useful when evaluating the acoustic impedance of isentropic nozzle and diffusers to subsequently study combustion instability in complex 3D combustion chambers, as in the work of Lamarque and Poinsot [27].

In the present study, a numerical approach is used to solve the quasi 1D LEE in the case where neither the total enthalpy nor the total entropy are constant over the entire domain. In the first section, the quasi 1D LEE system is presented. Compared to the system of equations exposed by [28], additional terms are added accounting for the compression or expansion stages in the system. Subsequently, analytical solutions are derived to validate the quasi 1D LEE numerical tool in two specific cases: isentropic compact nozzles [23] and a one dimensional compressor. Finally the reflection coefficient at the inlet air circuit of an helicopter combustion chamber is computed and used to evaluate the first two acoustic modes of a 3D combustion chamber. 


\section{The quasi 1D Linearized Euler Equations}

A quasi 1D Euler system of equations in the conservative form is presented (Nicoud and Wieczorek [28]). This set of equations states that the flow is strictly one-dimensional and therefore only changes over the stream direction $x$ are of relevance. It implies that only plane acoutic waves can be retrieved. The quasi 1D Euler system of equations reads:

$$
\begin{aligned}
\frac{\partial}{\partial t}(\rho A)+\frac{\partial}{\partial x}(\rho u A) & =0 \\
\frac{\partial}{\partial t}(\rho u A)+\frac{\partial}{\partial x}\left(\rho u^{2} A\right)+A \frac{\partial p}{\partial x}-A \mathcal{F} & =0 \\
\frac{\partial}{\partial t}\left(\rho h_{t} A\right)+\frac{\partial}{\partial x}\left(\rho u h_{t} A\right)-A \frac{\partial p}{\partial t}-A \dot{\mathcal{Q}}-A \mathcal{W} & =0
\end{aligned}
$$

where $\rho$ stands for the density of the fluid, $u$ the velocity in the $x$ direction, $p$ the thermodynamic pressure and $h_{t}$ the total enthalpy. In addition the variables $A, \dot{\mathcal{Q}}, \mathcal{F}$ and $\mathcal{W}$ stand for the crosssection area, an external energy source, the volume-specific force added by the compressor or added to the turbine, and the volume-specific work done by the compressor or supplied to the turbine, respectively. In the following the conservation of energy (3), which is function of the total enthalpy $h_{t}$, is replaced by the entropy $(s)$ equation:

$$
\rho T\left(\frac{\partial s}{\partial t}+u \frac{\partial s}{\partial x}\right)-\dot{\mathcal{Q}}=0
$$

All variables in Eqs. (1), (2) and (4) are decomposed in mean and fluctuating quantities $\left(\phi(x, t)=\bar{\phi}(x)+\phi^{\prime}(x, t)\right)$ and only first order terms are kept. Subsequently, harmonic perturbations are considered $\left(\phi^{\prime}=\hat{\phi} e^{-j \omega t}\right)$. The quasi 1D Linearized Euler Equations LEE for a calorically perfect gas read:

$$
\begin{gathered}
\left(\frac{\partial \bar{u}}{\partial x}+\bar{u} \frac{\partial}{\partial x}+\frac{\bar{u}}{A} \frac{\partial A}{\partial x}\right) \hat{\rho}+\left(\frac{\partial \bar{\rho}}{\partial x}+\bar{\rho} \frac{\partial}{\partial x}+\frac{\bar{\rho}}{A} \frac{\partial A}{\partial x}\right) \hat{u}-j \omega \hat{\rho}=0 \\
\left(\frac{1}{\bar{\rho}} \frac{\partial \bar{c}^{2}}{\partial x}+\frac{\bar{u}}{\bar{\rho}} \frac{\partial \bar{u}}{\partial x}+\frac{\bar{c}^{2}}{\bar{\rho}} \frac{\partial}{\partial x}\right) \hat{\rho}+\left(\frac{\partial \bar{u}}{\partial x}+\bar{u} \frac{\partial}{\partial x}\right) \hat{u}+(\gamma-1) \bar{T}\left(\frac{1}{\bar{p}} \frac{\partial \bar{p}}{\partial x}+\frac{\partial}{\partial x}\right) \hat{s}-\frac{\hat{\mathcal{F}}}{\bar{\rho}}-j \omega \hat{u}=0
\end{gathered}
$$




$$
\frac{\gamma r \overline{\mathcal{Q}}}{\bar{\rho} \bar{p}} \hat{\rho}+\frac{\partial \bar{s}}{\partial x} \hat{u}+\left(\bar{u} \frac{\partial}{\partial x}+(\gamma-1) \frac{\overline{\mathcal{Q}}}{\bar{p}}\right) \hat{s}-\frac{r}{\bar{p}} \hat{\mathcal{Q}}-j \omega \hat{s}=0
$$

where the linearized state equation and entropy expression (Eq. (8)) have been considered, i.e.

$$
\frac{\hat{p}}{\bar{p}}-\frac{\hat{\rho}}{\bar{\rho}}-\frac{\hat{T}}{\bar{T}}=0 \quad \text { and } \quad \frac{\hat{s}}{c_{p}}=\frac{\hat{p}}{\gamma \bar{p}}-\frac{\hat{\rho}}{\bar{\rho}} .
$$

This system of equations can be expressed as an algebraic linear system in the form

$$
\mathcal{A}\left[\begin{array}{c}
\hat{\rho} \\
\hat{u} \\
\hat{s}
\end{array}\right]=\left[\begin{array}{c}
0 \\
\frac{\hat{\mathcal{F}}}{\bar{\rho}} \\
\frac{r}{\bar{p}} \hat{\mathcal{Q}}
\end{array}\right]
$$

where

$$
\mathcal{A}=\left[\begin{array}{ccc}
\frac{\partial \bar{u}}{\partial x}+\bar{u} \frac{\partial}{\partial x}+\frac{\bar{u}}{A} \frac{\partial A}{\partial x}-j \omega & \frac{\partial \bar{\rho}}{\partial x}+\bar{\rho} \frac{\partial}{\partial x}+\frac{\bar{\rho}}{A} \frac{\partial A}{\partial x} & 0 \\
\frac{1}{\bar{\rho}} \frac{\partial \bar{c}^{2}}{\partial x}+\frac{\bar{u}}{\bar{\rho}} \frac{\partial \bar{u}}{\partial x}+\frac{\bar{c}^{2}}{\bar{\rho}} \frac{\partial}{\partial x} & \frac{\partial \bar{u}}{\partial x}+\bar{u} \frac{\partial}{\partial x}-j \omega & (\gamma-1) \bar{T}\left(\frac{1}{\bar{p}} \frac{\partial \bar{p}}{\partial x}+\frac{\partial}{\partial x}\right) \\
\frac{\gamma r \overline{\mathcal{Q}}}{\bar{\rho} \bar{p}} & \frac{\partial \bar{s}}{\partial x} & \bar{u} \frac{\partial}{\partial x}+(\gamma-1) \frac{\overline{\mathcal{Q}}}{\bar{p}}-j \omega
\end{array}\right]
$$

Note that this system of linearized equations models propagation of plane acoustic waves through a calorically perfect medium. These expressions are, nevertheless, very suitable when modeling the upstream or downstream region of typical aeronautical combustion chambers. Combustion instabilitiy, a phenomenon highly dependent on boundary conditions, takes place generally at low frequencies, i.e. at frequencies where only plane acoustic waves are present. Moreover, under normal operating conditions, reactions only takes place within the confinement of a combustion chamber. Accordingly, assuming a constant heat capacity $c_{p}$ either upstream or downstream of the combustion chamber is fairly correct. Note however that the assumption can be easily relaxed as far as Eq. (9) is solved numerically.

Within the computational domain, the Quasi-1D LEE (Eqs. 5, 6 and 7) are discretized by finite differences (FD) with a second order centered scheme. At the inlet, a first order FD downwind scheme is applied, whereas at the outlet a first order FD upwind scheme is used. A staggered grid 
arrangement (velocity fluctuations stored at the cell edges, density and entropy fluctuations stored at the cell centers as shown in Fig. 1.) has been used in order to avoid the pressure field to be contaminated by the classical odd-even decoupling phenomenon [29, 30].

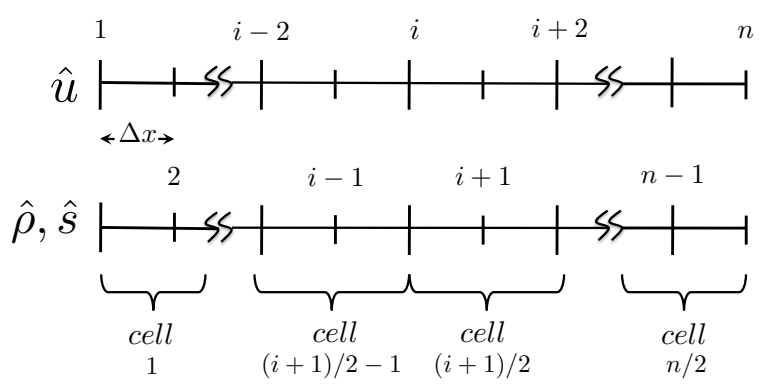

Fig. 1 Staggered grid

For the $(i+1) / 2$ cell, equations of continuity, momentum and entropy are discretized as follows:

- continuity equation (node $i+1$ )

$$
\begin{array}{r}
\hat{\rho}_{i+1} \frac{\partial \bar{u}_{i+1}}{\partial x}+\bar{u}_{i+1} \frac{\left(\hat{\rho}_{i+3}-\hat{\rho}_{i-1}\right)}{4 \Delta x}+\bar{\rho}_{i+1} \frac{\left(\hat{u}_{i+2}-\hat{u}_{i}\right)}{2 \Delta x}+\frac{\left(\hat{u}_{i+2}+\hat{u}_{i}\right)}{2} \frac{\partial \bar{\rho}_{i+1}}{\partial x}+\frac{\left(\hat{u}_{i+2}+\hat{u}_{i}\right)}{2} \frac{\bar{\rho}_{i+1}}{A_{i+1}} \frac{\partial A_{i+1}}{\partial x}+ \\
\hat{\rho}_{i+1} \frac{\bar{u}_{i+1}}{A_{i+1}} \frac{\partial A_{i+1}}{\partial x}-j \omega \hat{\rho}_{i+1}=0
\end{array}
$$

- momentum equation (node $i$ )

$$
\begin{array}{r}
\frac{\left(\hat{\rho}_{i+1}+\hat{\rho}_{i-1}\right)}{2 \bar{\rho}_{i}} \frac{\partial \bar{c}_{i}^{2}}{\partial x}+\frac{\left(\hat{\rho}_{i+1}+\hat{\rho}_{i-1}\right) \bar{u}_{i}}{2 \bar{\rho}_{i}} \frac{\partial \bar{u}_{i}}{\partial x}+\frac{\bar{c}_{i}^{2}}{\bar{\rho}_{i}} \frac{\left(\hat{\rho}_{i+1}-\hat{\rho}_{i-1}\right)}{2 \Delta x}+\hat{u}_{i} \frac{\partial \bar{u}_{i}}{\partial x}+\bar{u}_{i, 1} \frac{\left(\hat{u}_{i+2}-\hat{u}_{i-2}\right)}{4 \Delta x}+ \\
(\gamma-1) \frac{\bar{T}_{i}\left(\hat{s}_{i+1}+\hat{s}_{i-1}\right)}{2 \bar{p}_{i}} \frac{\partial \bar{p}_{i}}{\partial x}+(\gamma-1) \bar{T}_{i} \frac{\left(\hat{s}_{i+1}-\hat{s}_{i-1}\right)}{2 \Delta x}-j \omega \hat{u}_{i}=\frac{\hat{\mathcal{F}}_{i}}{\bar{\rho}_{i}}
\end{array}
$$

- entropy equation(node $i+1)$

$$
\begin{aligned}
\frac{\gamma r \bar{q}_{i+1}}{\bar{\rho}_{i+1} \bar{p}_{i+1}} \hat{\rho}_{i+1}+\frac{\left(\hat{u}_{i+2}+\hat{u}_{i}\right)}{2} \frac{\partial \bar{s}_{i+1}}{\partial x}+\bar{u}_{i+1} \frac{\left(\hat{s}_{i+3}-\hat{s}_{i-1}\right)}{4 \Delta x}+(\gamma-1) \frac{\bar{q}_{i+1}}{\bar{p}_{i+1}} \hat{s}_{i+1} & -\frac{r}{\bar{p}_{i+1}} \hat{q}_{i+1} \hat{s}_{i+1}-j \omega \hat{s}_{i+1} \\
& =\hat{\dot{Q}}_{i+1}
\end{aligned}
$$


The resulting discretized matrix $\mathbb{A}$ for the previous system of equations is a sparse rectangular matrix of size $m \times m$ size, in which $m=3 n / 2$ and $n / 2$ is the number of cells of the system. A Jacobi preconditioner is used to improve the conditioning of matrix $\mathbb{A}$ and the linear system is solved by inverting the preconditioned matrix through the $L U$ factorization. The resulting numerical tool is termed 'SNozzle'.

\section{Definition of waves}

In the frequency domain, acoustic and entropy waves $\hat{w}$ are defined by an amplitude $B$ and a phase $\phi$, so that $\hat{w}=B e^{\boldsymbol{j} \phi}$. They read $[25,31]$

$$
\begin{aligned}
& \hat{w}^{+}=B^{+} e^{j \omega x / \bar{c}(1+\overline{\mathcal{M}})}=\frac{\hat{p}}{\gamma \bar{p}}+\frac{\hat{u}}{\bar{c}} \\
& \hat{w}^{-}=B^{-} e^{-j \omega x / \bar{c}(1-\overline{\mathcal{M}})}=\frac{\hat{p}}{\gamma \bar{p}}-\frac{\hat{u}}{\bar{c}} \\
& \hat{w}^{S}=B^{S} e^{j \omega x / \bar{c} \overline{\mathcal{M}}}=\frac{\hat{s}}{c_{p}}
\end{aligned}
$$

where $\hat{w}^{+}$and $\hat{w}^{-}$represent the non-dimensional acoustic waves propagating respectively in the direction of the flow at speed $(\bar{u}+\bar{c})$, and against the flow at speed $(\bar{u}-\bar{c}) \cdot \hat{w}^{S}$ stands for the entropy wave that is convected at the mean flow velocity $(\bar{u})$. Inversely, the variables $\hat{p}$ and $\hat{u}$ are expressed in terms of acoustic waves as

$$
\frac{\hat{p}}{\gamma \bar{p}}=\frac{1}{2}\left(\hat{w}^{+}+\hat{w}^{-}\right) \quad \text { and } \quad \frac{\hat{u}}{\bar{c}}=\frac{1}{2}\left(\hat{w}^{+}-\hat{w}^{-}\right)
$$

The waves $\hat{w}$, that can appear in a $1 \mathrm{D}$ system, are shown in Fig. (2). Indices 1 and 2 identify the upstream and downstream state respectively.

$$
\begin{aligned}
& \hline R_{A A} \rightarrow \hat{w}_{1}^{-} / \hat{w}_{1}^{+} \rightarrow \text { with } \hat{w}_{1}^{S}=0 \\
& R_{S A} \rightarrow \hat{w}_{1}^{-} / \hat{w}_{1}^{S} \rightarrow \text { with } \hat{w}_{1}^{+}=0 \\
& T_{A A} \rightarrow \hat{w}_{2}^{+} / \hat{w}_{1}^{+} \rightarrow \text { with } \hat{w}_{1}^{S}=0 \\
& T_{S A} \rightarrow \hat{w}_{2}^{+} / \hat{w}_{1}^{S} \rightarrow \text { with } \hat{w}_{1}^{+}=0 \\
& \hline
\end{aligned}
$$

Table 1 Wave coefficients definition 


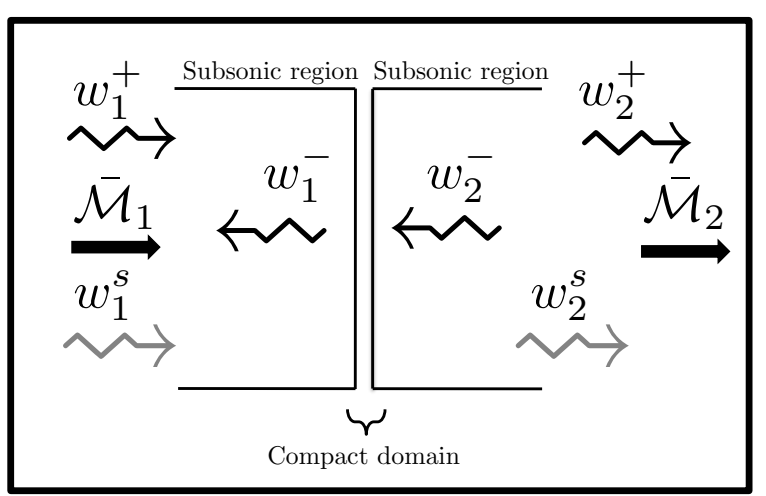

(a)

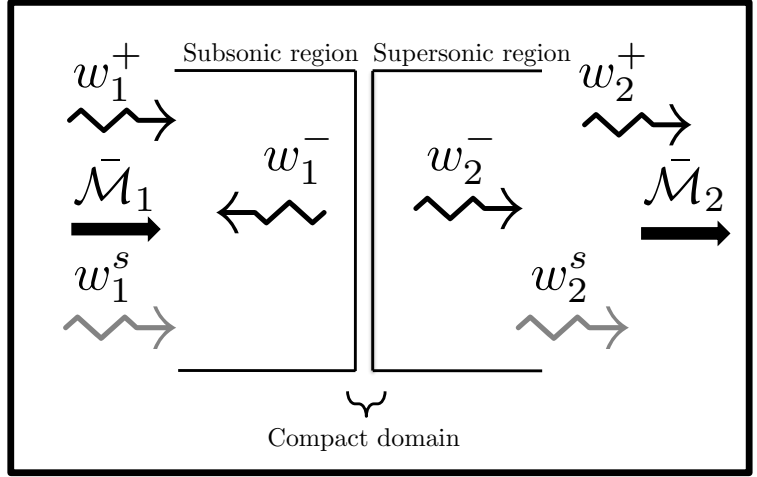

(b)

Fig. 2 Configurations under the compact assumption. (a) subsonic-subsonic. (b) subsonicsupersonic.

The reflection $R$ and transmission $T$ coefficients are now defined as shown in table 1 . The coefficient $R_{A A}$ is the reflection coefficient that measures the amplitude of the outgoing upstream traveling acoustic wave $\hat{w}_{1}^{-}$with respect to an incoming downstream traveling wave $\hat{w}_{1}^{+}$when no incoming entropy waves are assumed present $\left(\hat{w}_{1}^{S}=0\right)$. Similarly $R_{S A}$ relates the amplitude of an upstream outgoing traveling acoustic wave $\hat{w}_{1}^{-}$with respect to an incoming downstream traveling entropy wave $\hat{w}_{1}^{S}$. In this case, no incoming acoustic waves are present $\left(\hat{w}_{1}^{+}=0\right)$. The transmitted waves, defined by the tranmission coefficient $T$, are determined in a similar way. Being these acoustic parameters defined for a downstream boundary condition, they are used to characterize systems such as nozzles or turbines. The definition of these parameters should be inverted if an upstream boundary condition is taken as the reference, which is the case for intakes and compressors.

In the next two sections attention is paid to two of these cases. First, nozzles are treated as isentropic systems in which no enthalpy expansion is present, i.e. no turbines stages are considered. In this manner, analytical solutions found in the literature for the acoustic response of compact nozzles to incoming acoustic or entropy waves [23] can be compared to the solution of the quasi 1D LEE in the zero frequency limit $(\omega \approx 0)$. Second, the acoustic response of an ideal compact compressor embedded in a constant section duct is evaluated. This simplified case permits to compare the results obtained by the quasi 1D LEE to the solutions given by analytical relations which are indeed a generalization of analytical models such as the one exposed in [4, 23]. 


\section{Response of isentropic nozzles}

As already stated in the introductory part of this work, determining appropriate acoustic boundary conditions for combustion chambers is crucial in order to correctly predict combustion instabilities. These acoustic boundary conditions depend, among others, on the mean flow either upstream (compressor stages) or downstream (turbine stages). As a first approximation, it is useful to consider the flow in these regions as isentropic $(\overline{\mathcal{Q}}, \hat{\mathcal{Q}}=0)$ and isenthalpic $(\overline{\mathcal{F}}, \hat{\mathcal{F}}=0)$. This means that the combustion chamber is only surrounded by perfect diffusers and nozzles.

\section{A. Compact Nozzles}

The continuity and energy relations in the framework of the 1D linearized Euler equations for compact systems (see Eqs. (62) and (64) in appendix) reduce to:

$$
\begin{gathered}
\frac{\hat{\rho}_{1}}{\bar{\rho}_{1}}+\frac{\hat{u}_{1}}{\bar{u}_{1}}=\frac{\hat{\rho}_{2}}{\bar{\rho}_{2}}+\frac{\hat{u}_{2}}{\bar{u}_{2}} \\
\frac{\hat{T}_{t 1}}{\bar{T}_{t 1}}=\frac{\hat{T}_{t 2}}{\bar{T}_{t 2}}
\end{gathered}
$$

in which it has been assumed that the fluid has a constant specific heat $c_{p}$ and therefore $\hat{h}=c_{p} \hat{T}$. Expressing the instantaneous total temperature as $T_{t}=T\left(1+[(\gamma-1) / 2] \mathcal{M}^{2}\right)$, in which $\mathcal{M}=u / c$ represents the instantaneous Mach number, the following relation results

$$
\frac{\hat{T}_{t}}{\bar{T}_{t}}=\frac{\hat{T}}{\bar{T}}+\frac{(\gamma-1) \overline{\mathcal{M}} \hat{\mathcal{M}}}{1+\frac{\gamma-1}{2} \overline{\mathcal{M}}^{2}}=\frac{\hat{T}}{\bar{T}}+\frac{(\gamma-1)}{1+\frac{\gamma-1}{2} \overline{\mathcal{M}}^{2}}\left(\overline{\mathcal{M}}^{2} \frac{\hat{u}}{\bar{u}}-\overline{\mathcal{M}}^{2} \frac{\hat{c}}{\bar{c}}\right)
$$

where $\hat{c} / \bar{c}=\hat{T} / 2 \bar{T}$. Replacing Eq. (20) in Eq. (19) and considering also the relations exposed in Eq. (8), the following expression yields:

$$
\frac{1}{1+\frac{\gamma-1}{2} \overline{\mathcal{M}}_{1}^{2}}\left(\overline{\mathcal{M}}_{1} \frac{\hat{u}_{1}}{\bar{c}_{1}}+\frac{\hat{\rho}_{1}}{\bar{\rho}_{1}}+\frac{\gamma}{(\gamma-1)} \frac{\hat{s}_{1}}{c_{p}}\right)=\frac{1}{1+\frac{\gamma-1}{2} \overline{\mathcal{M}}_{2}{ }^{2}}\left(\overline{\mathcal{M}}_{2} \frac{\hat{u}_{2}}{\bar{c}_{2}}+\frac{\hat{\rho}_{2}}{\bar{\rho}_{2}}+\frac{\gamma}{(\gamma-1)} \frac{\hat{s}_{2}}{c_{p}}\right)
$$

Finally, substituting the definition all variables into a wave description (Eq. (17)) at $x=0$ into Eqs. (18) and (21) results in: 


$$
\begin{aligned}
\frac{1}{2} \frac{\left(1+\overline{\mathcal{M}}_{1}\right)}{\overline{\mathcal{M}}_{1}} \hat{w}_{1}^{+}-\frac{1}{2} \frac{\left(1-\overline{\mathcal{M}}_{1}\right)}{\overline{\mathcal{M}}_{1}} \hat{w}_{1}^{-}+\hat{w}_{1}^{S} & =\frac{1}{2} \frac{\left(1+\overline{\mathcal{M}}_{2}\right)}{\overline{\mathcal{M}}_{2}} \hat{w}_{2}^{+}-\frac{1}{2} \frac{\left(1-\overline{\mathcal{M}}_{2}\right)}{\overline{\mathcal{M}}_{2}} \hat{w}_{2}^{-}+\hat{w}_{2}^{S} \\
\frac{1}{2} \frac{\left(1+\overline{\mathcal{M}}_{1}\right)}{\zeta_{1}} \hat{w}_{1}^{+}+\frac{1}{2} \frac{\left(1-\overline{\mathcal{M}}_{1}\right)}{\zeta_{1}} \hat{w}_{1}^{-}+\frac{1}{\zeta_{1}(\gamma-1)} \hat{w}_{1}^{S} & =\frac{1}{2} \frac{\left(1+\overline{\mathcal{M}}_{2}\right)}{\zeta_{2}} \hat{w}_{2}^{+}+\frac{1}{2} \frac{\left(1-\overline{\mathcal{M}}_{2}\right)}{\zeta_{2}} \hat{w}_{2}^{-}+\frac{1}{\zeta_{2}(\gamma-1)} \hat{w}_{2}^{S}
\end{aligned}
$$

in which the notation $\zeta=1+[(\gamma-1) / 2] \overline{\mathcal{M}}^{2}$ has been introduced.

Now the analytical relations concerning the acoustic response of compact nozzles [23, 31] are recalled. An unchoked nozzle, which is characterized by a subsonic flow everywhere, needs to be acoustically constrained both at the inlet and at the outlet. In order to acoustically characterize the nozzle independently from boundary conditions, the outlet is fixed as totally non-reflecting $\left(\hat{w}_{2}^{-}=0\right)$. Note that this procedure also simplifies the final mathematical expressions. If the acoustic wave $\hat{w}_{2}^{-}$ is of relevance, it can be considered in the equations through a reflection coefficient $R=\hat{w}_{2}^{-} / \hat{w}_{2}^{+}$. Particularly, an expression for $R_{A A}$ for an arbitrary value of $R=\hat{w}_{2}^{-} / \hat{w}_{2}^{+}$can be found in the work of Lamarque and Poinsot [27]. Since neither heat nor work is added to the system, entropy waves remain constant along the nozzle $\left(\hat{w}_{1}^{S}=\hat{w}_{2}^{S}\right)$. Eqs. $(22)$ and $(23)$ are combined in order to obtain the following expressions:

$$
\begin{gathered}
R_{A A}=\left(\frac{\overline{\mathcal{M}}_{1}-\overline{\mathcal{M}}_{2}}{\overline{\mathcal{M}}_{1}+\overline{\mathcal{M}}_{2}}\right)\left(\frac{\overline{\mathcal{M}}_{1}+1}{\overline{\mathcal{M}}_{1}-1}\right)\left(\frac{1-\frac{1}{2}(\gamma-1) \overline{\mathcal{M}}_{1} \overline{\mathcal{M}}_{2}}{1+\frac{1}{2}(\gamma-1) \overline{\mathcal{M}}_{1} \overline{\mathcal{M}}_{2}}\right) \quad \text { with } \quad \hat{w}_{1}^{S}, \hat{w}_{2}^{-}=0 \\
R_{S A}=-\left(\frac{\overline{\mathcal{M}}_{2}-\overline{\mathcal{M}}_{1}}{1-\overline{\mathcal{M}}_{1}}\right)\left(\frac{\overline{\mathcal{M}}_{1}}{1+\frac{1}{2}(\gamma-1) \overline{\mathcal{M}}_{1} \overline{\mathcal{M}}_{2}}\right) \quad \text { with } \quad \hat{w}_{1}^{+}, \hat{w}_{2}^{-}=0 \\
T_{A A}=\left(\frac{2 \overline{\mathcal{M}}_{2}}{1+\overline{\mathcal{M}}_{2}}\right)\left(\frac{1+\overline{\mathcal{M}}_{1}}{\overline{\mathcal{M}}_{1}+\overline{\mathcal{M}}_{2}}\right)\left(\frac{1+\frac{1}{2}(\gamma-1) \overline{\mathcal{M}}_{2}^{2}}{1+\frac{1}{2}(\gamma-1) \overline{\mathcal{M}}_{1} \overline{\mathcal{M}}_{2}}\right) \quad \text { with } \quad \hat{w}_{1}^{S}, \hat{w}_{2}^{-}=0 \\
T_{S A}=\left(\frac{\overline{\mathcal{M}}_{2}-\overline{\mathcal{M}}_{1}}{1+\overline{\mathcal{M}}_{2}}\right)\left(\frac{\overline{\mathcal{M}}_{2}}{1+\frac{1}{2}(\gamma-1) \overline{\mathcal{M}}_{1} \overline{\mathcal{M}}_{2}}\right) \quad \text { with } \quad \hat{w}_{1}^{+}, \hat{w}_{2}^{-}=0
\end{gathered}
$$

In a choked nozzle, when assuming the outlet pressure low enough, the flow becomes supersonic downstream the throat. In this case only acoustic boundary conditions at the inlet of the system 
should be imposed. Imposing acoustic boundary conditions at the outlet leads to an ill-posed problem: setting a given reflecting condition (for instance $\left(\hat{w}_{2}^{-}=0\right)$ ) is physically not correct. In order to find the different wave coefficients shown in table 1, the problem is divided into two. First, the subsonic region is solved. Accordingly, the throat of the nozzle is established as the outlet with $\overline{\mathcal{M}}_{2}=1$. Second, the entire domain is solved by accounting for the results obtained in the first step. The final coefficients are then obtained yielding:

$$
\begin{aligned}
& R_{A A}=\frac{1-\frac{1}{2}(\gamma-1) \overline{\mathcal{M}}_{1}}{1+\frac{1}{2}(\gamma-1) \overline{\mathcal{M}}_{1}} \quad \text { with } \quad \hat{w}_{1}^{S}=0 \\
& R_{S A}=\frac{-\overline{\mathcal{M}}_{1}}{1+\frac{1}{2}(\gamma-1) \overline{\mathcal{M}}_{1}} \quad \text { with } \quad \hat{w}_{1}^{+}=0 \\
& T_{A A}=\frac{1+\frac{1}{2}(\gamma-1) \overline{\mathcal{M}}_{2}}{1+\frac{1}{2}(\gamma-1) \overline{\mathcal{M}}_{1}} \quad \text { with } \quad \hat{w}_{1}^{S}=0 \\
& T_{S A}=\frac{\frac{1}{2}\left(\overline{\mathcal{M}}_{2}-\overline{\mathcal{M}}_{1}\right)}{1+\frac{1}{2}(\gamma-1) \overline{\mathcal{M}}_{1}} \quad \text { with } \quad \hat{w}_{1}^{+}=0
\end{aligned}
$$

Equations (24) to (31) are classical equations for compact nozzles, first encountered in the work of Marble and Candel [23]. It is interesting to observe that the coefficients $R_{A A}$ and $R_{S A}$ do not depend on the Mach number $\overline{\mathcal{M}}_{2}$ at the supersonic outlet. Indeed, as expected, they only depend on $\overline{\mathcal{M}}_{1}$ and on the flow conditions at the the throat, where $\overline{\mathcal{M}}=1$.

\section{B. Non compact nozzles}

The compact assumption is very useful to study the acoustic response of a nozzle when the acoustic wave length $\lambda$ is much larger than the nozzle length. Nevertheless, when acoustic and entropy disturbances fluctuate at large frequencies the jump conditions showed previously become unsuited since they do not account for phase changes across the nozzle. In order to study the influence of 'non-compactness', different methods are found in the literature [23-25, 32]. Marble and Candel [23] stated that the main influence of the nozzle finiteness is to alter the phase between the two wave trains. They showed it analytically by considering that the mean flow velocity changes linearly through the nozzle. Nevertheless, this assumption is too restrictive for real systems. In this 
section, the response of non compact nozzles is computed by solving numerically the Linearized Euler Equations under the quasi-1D assumption (see section II).

Two different nozzle configurations are studied. They are shown in Figure 3 and the mean flow and geometrical parameters are given in table 2 .

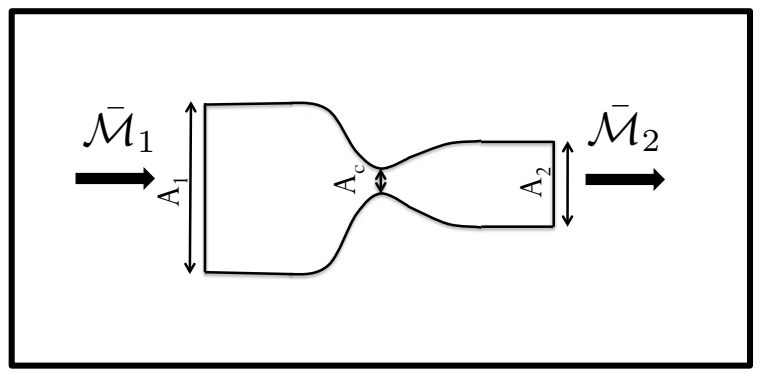

(a)

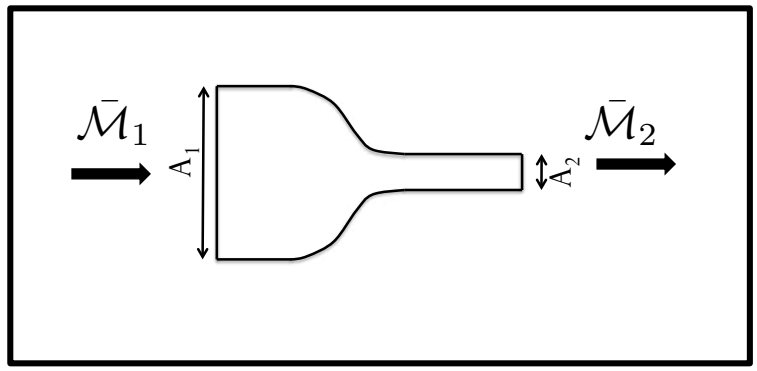

(b)

Fig. 3 Choked and Unchoked configurations studied

\begin{tabular}{|c|c|c|}
\hline & $\begin{array}{c}\text { Unchoked } \\
\text { Case }\end{array}$ & $\begin{array}{c}\text { Choked } \\
\text { Case }\end{array}$ \\
\hline$A_{1} / A_{c}$ & 5.651 & 5.873 \\
\hline$A_{2} / A_{c}$ & 1.000 & 1.032 \\
\hline$\overline{\mathcal{M}}_{2}$ & 0.8 & 1.2 \\
\hline$\overline{\mathcal{M}}_{1}$ & \multicolumn{2}{|c|}{0.1} \\
\hline $\bar{T}_{1}$ & \multicolumn{2}{|c|}{$1300 \mathrm{~K}$} \\
\hline $\bar{p}_{1}$ & \multicolumn{2}{|c|}{$800 \mathrm{kPa}$} \\
\hline
\end{tabular}

Table 2 Mean flow inlet conditions and geometrical parameters

In order to test the reliability of the SNozzle tool (see section II), the results given by this solver are compared to those obtained by solving the complete set of Euler Equations using a numerical solver for the unsteady fluid flow equations. Besides, they are compared to the analytical results obtained under the compact assumption. More precisely, the following approaches are compared in what follows:

- Analytics: The acoustic parameters $R_{A A}, R_{S A}, T_{A A}, T_{S A}$ are computed through the relations proposed by Marble \& Candel [23]. Equations (24) to (27), and (28) to (31) are then used.

- Quasi-1D LEE (SNozzle): The inlet Mach number $\overline{\mathcal{M}}_{1}$, temperature $\bar{T}_{1}$ and pressure $\bar{p}_{1}$ 
are given as inputs to SNozzle as well as the geometry ( $\mathrm{x}$ coordinate and variable section area $A)$. The outlet is taken as totally non reflecting $\left(\hat{w}_{2}^{-}=0\right)$ for the non-choked case and is simply non constrained for the choked case. At the inlet either $\hat{w}_{1}^{+}$or $\hat{w}^{S}$ are imposed. Two thousand points are used for both geometries. Note that it was checked that grid convergence is reached for this level of spatial resolution up to a reduced frequency $\Omega=1$. Results were validated against the analytical quasi-1D solver proposed in the work of Duran and Moreau $[26]$.

- The 3D Euler Equations (AVBP): The LES solver AVBP [33] is used, in which the viscous terms of the Navier Stokes equations have been neglected. This numerical tool, developed at CERFACS, is second order accurate in space and time. The Euler equations are spatially discretized under the finite volume formulation with a cell-vertex approach. Care has to be taken specially with the use of the inlet/outlet boundary conditions, since both the mean flow and either the entropy or acoustic wave must be imposed [34]. Special attention should be paid on how these waves are introduced into the problem since, on the one hand, a totally non-reflecting inlet/outlet are sought (the imposed and reflected waves, $\hat{w}_{1}^{+}$and $\hat{w}_{1}^{-}$, should be completely evacuated) and, on the other hand, the mean flow must remain constant. In AVBP, a NSCBC approach [35] is used for the treatment of waves crossing the boundaries.

\section{Results}

The value of both transmission and reflection coefficients are shown in Fig. (4). They are plotted against the non-dimensional frequency $\Omega=f l_{n} / \bar{c}_{1}$ where $f$ is the frequency, $l_{n}$ the length of the nozzle and $\bar{c}_{1}$ the speed of sound at the inlet. It is observed that analytical results are well suited when considering small frequencies $(\Omega \rightarrow 0)$. Both numerical tools converge to Marble \& Candel expressions when $\lambda \gg l_{n}$ for all coefficients.

At small frequencies, it is observed that the main influence of a choked nozzle is the increase of noise $\left(\hat{w}_{2}^{+} \nearrow\right)$ due to entropy waves, as already stated in [31]. This can be seen in the value of $T_{S A}$ $\left(\hat{w}_{2}^{+} / \hat{w}_{1}^{S}\right)$ which is as twice as large when the nozzle is choked. The reflected wave $\hat{w}_{1}^{-}$also increases, but to a lesser extent. On the other hand, the acoustic response of the nozzle caused by an incoming 


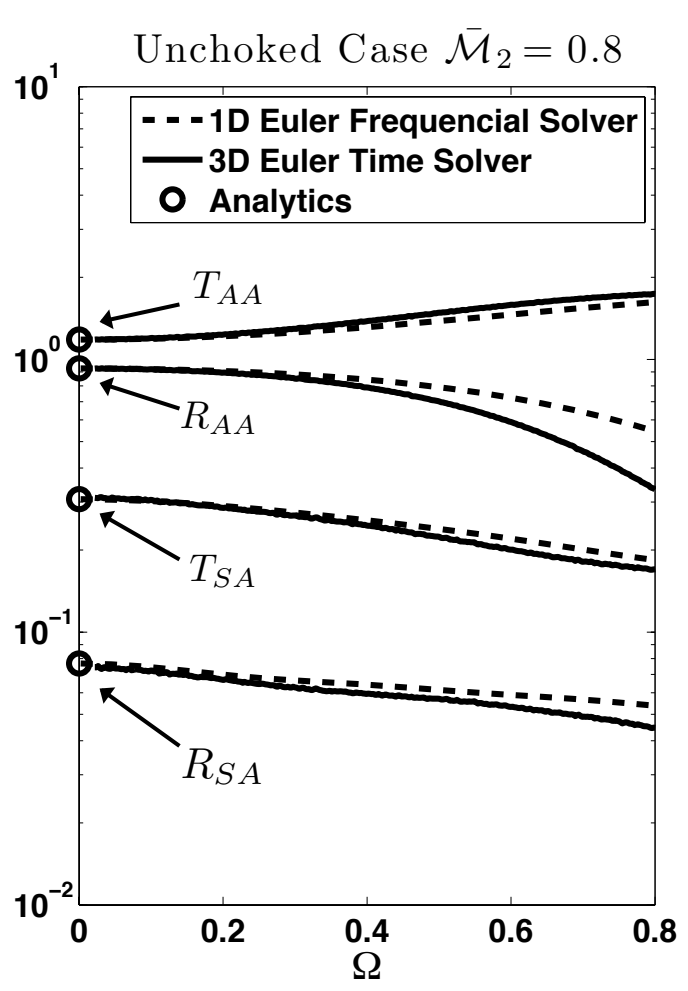

(a)

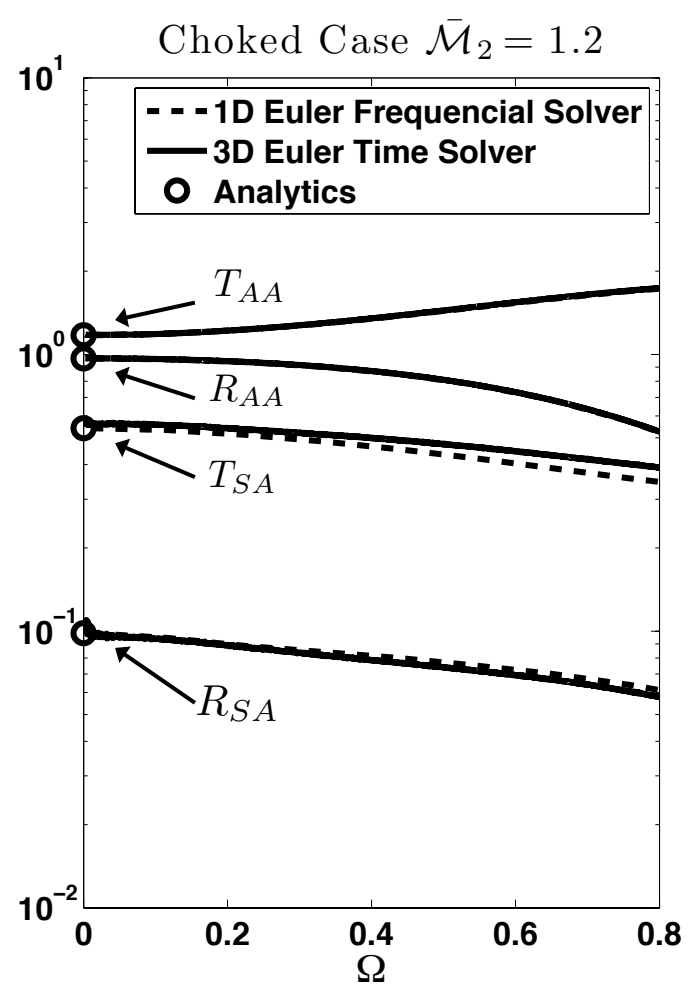

(b)

Fig. 4 Reflection and Transmission coefficients for both unchoked and choked cases

acoustic wave remains quite similar $\left(T_{A A}\right.$ and $\left.R_{A A}\right)$ for both unchoked and choked configurations.

When the reduced frequency increases, the value of the acoustic coefficients starts to move away smoothly from the analytical results. On the one hand, when taking into account the unchoked case, it can be observed that results coming from both numerical solvers can be considered similar up to $\Omega \approx 0.2$. For larger frequencies, the $3 \mathrm{D}$ Euler solver curves representing $R_{A A}, T_{S A}$ and $R_{S A}$ start to decrease faster, the largest differences appearing for $R_{A A}$. On the other hand both numerical solvers give similar results when considering the choked case for the values of $\Omega$ under consideration. It is interesting to notice that AVBP, a numerical tool used in this study to solve the 3D Euler equations with second order of accuracy in space and time, is able to retrieve quite well the acoustic response of a chocked nozzle whereas underestimates the acoustic reflection $R_{A A}$ occurring in an unchoked nozzle. This is probably due to the numerical scheme used in the 3D Euler solver, which appears to be dissipative for small length waves, which is the case for the downstream travelling waves $\hat{w}_{1}^{S}$ and $\hat{w}_{1}^{+}$in an unchoked nozzle for high values of $\Omega$. Recall that the mesh is kept unchanged when 
$\Omega$ increases, so that numerical errors increase.

\section{Response of an ideal compressor}

In the previous sections, focus was made on the acoustic response of ducts with varying sections causing a change in the mean flow: the nozzle case. The purpose of this section is to focus on the acoustic response of a system when the changes in the mean flow are caused by enthalpy jumps. An ideal compact compressor is considered here. The first step when modeling a compressor is to consider it as an element that creates a difference in both the total pressure and the kinetic energy in the flow, i.e., an element that exerts a work on the flow by changing its total enthalpy. As represented in Fig. 5, a jump of total enthalpy is considered in the middle of the constant section duct. This jump comes from the characteristic curve of the turbo-machinery.

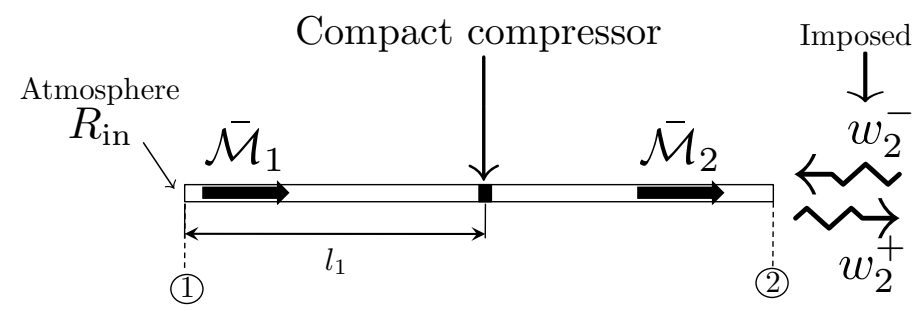

Fig. 5 Scheme of the compact compressor configuration

In this configuration an upstream traveling acoustic wave $\hat{w}_{2}^{-}$is imposed at the outlet while an acoustic reflection $R_{\text {in }}$ is imposed at the inlet. This analytical/numerical setup is a simplified representation of the inlet air circuit and compressor of an aeronautical engine. The acoustic waves, produced in the combustion chamber, travel upstream through the inlet air circuit and the compact compressor until reaching the atmosphere which is considered as a partial reflecting acoustic condition. A downstream acoustic traveling wave is subsequently reflected and propagates all the way through the inlet air circuit and compact compressor until arriving at the inlet of the combustion chamber. The acoustic reflection coefficient $R_{\text {out }}=\hat{w}_{2}^{+} / \hat{w}_{2}^{-}$is then estimated. As done before, this study is carried out both analytically and numerically (SNozzle).

It should be noted that, although not explicited in this paper, a similar methodology can be applied for the acoustic response of a system composed by several turbines stages and a nozzle. 
Yet, some simplifications in the acoustic model can be made when evaluating the impedance at the combustion chamber outlet. Indeed, the flow is generally choked at the first high pressure turbine stage [36]. In such a case only the upstream flow influences the acoustic impedance at the outlet of the combustion chamber. Moreover, a simple expression for the acoustic impedance of such a boundary condition can be retrieved analytically in the low frequency limit [27]. A stronger simplification and still satisfactory model can be made as well if acoustics of this choked outlet is seen as a wall: an acoustic boundary where perturbations of velocity are not allowed.

\section{A. Modeling of the compact compressor element}

The compressor may be seen as an element that exerts a change in the total enthalpy of the flow. In a two dimensional model, this jump of total enthalpy through the compressor can be properly modeled by the conservation of rothalpy $I$ as follows:

$$
I=h_{t, 1}-U_{1} v_{\theta, 1}=h_{t, 2}-U_{2} v_{\theta, 2} \quad \text { or } \quad h_{t, 1}-h_{t, 2}=U_{1} v_{\theta, 1}-U_{2} v_{\theta, 2}
$$

where $U$ is the angular velocity of the compressor and $v_{\theta}$ is the projection of the velocity vector $\boldsymbol{v}$ on the plane of $U$. In a one dimensional model, however, tangential velocities $v_{\theta}$, which are normal to the flow stream $u$, do not count among the unknowns and cannot be used to model the rotor effect. The modeling strategy carried out in this work relies on the characteristic curves, i.e. the compressor map, which relates the total pressure ratio $\pi_{c}$ to the mass flow $\dot{m}$ [37] as shown in Fig. 6 .

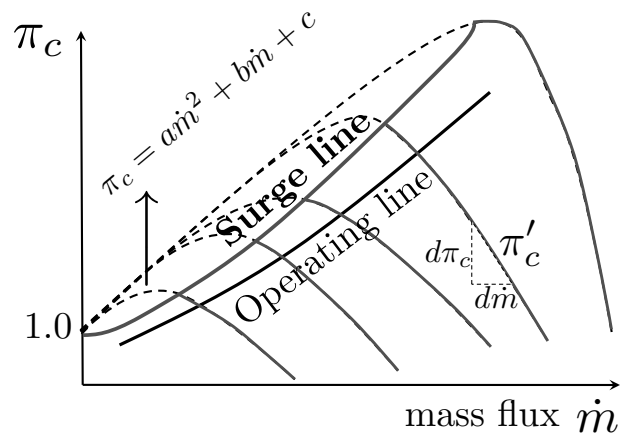

Fig. 6 Map of a typical compressor

The total enthalpy ratio is then given by: 


$$
\frac{h_{t, 2}}{h_{t, 1}}=\frac{T_{t, 2}}{T_{t, 1}}=\pi_{T} \quad \text { where } \quad \pi_{T}=\pi_{c}^{(\gamma-1) / \eta_{p} \gamma}
$$

where $\pi_{T}$ and $\eta_{p}$ stand for the total temperature ratio and the polytropic efficiency respectively. The total pressure ratio $\pi_{c}$ is defined by a second order polynomial $\pi_{c}=a \dot{m}^{2}+b \dot{m}+c$ and clearly depends on the performance characteristics of the compressor. The logarithmic derivative of $T_{t}$ can be then computed as:

$$
\frac{\eta_{p} \gamma}{\gamma-1}\left(\frac{\hat{T}_{t, 2}}{\bar{T}_{t, 2}}-\frac{\hat{T}_{t, 1}}{\bar{T}_{t, 1}}\right)=\frac{\hat{\pi}_{c}}{\bar{\pi}_{c}} \quad \text { where } \quad \frac{\hat{\pi}_{c}}{\bar{\pi}_{c}}=\frac{2 a \overline{\dot{m}}^{2}+b \overline{\dot{m}}}{a \bar{m}^{2}+b \dot{\dot{m}}+c}\left(\frac{\hat{\dot{m}}}{\overline{\dot{m}}}\right)=K \frac{\hat{\dot{m}}}{\dot{\dot{m}}} \quad \text { and } \quad \overline{\dot{m}}=\bar{\rho} \bar{u} A
$$

Adding a compact one dimensional compressor to the system is therefore equivalent to add an additional term to the left hand side of the energy equation (19), as follows :

$$
\frac{\hat{T}_{t, 1}}{\bar{T}_{t, 1}}+\frac{\gamma-1}{\eta_{p} \gamma} K\left(\frac{\hat{\rho}_{1}}{\bar{\rho}_{1}}+\frac{\hat{u}_{1}}{\bar{u}_{1}}\right)=\frac{\hat{T}_{t, 2}}{\bar{T}_{t, 2}}
$$

in which the constant $K$ characterizes the compressor at a given operating point. As function of the Mach number $\overline{\mathcal{M}}$ and the frequency $\omega$ of the acoustic perturbations, the continuity (Eq. (18)) and energy (Eq. (35)) equations read respectively:

$$
\begin{gathered}
\frac{\left(1+\overline{\mathcal{M}}_{1}\right)}{\overline{\mathcal{M}}_{1}} B_{1}^{+} e^{j \omega x / \bar{c}_{1}\left(1+\bar{M}_{1}\right)}-\frac{\left(1-\overline{\mathcal{M}}_{1}\right)}{\overline{\mathcal{M}}_{1}} B_{1}^{-} e^{-j \omega x / \bar{c}_{1}\left(1-\bar{M}_{1}\right)}= \\
\frac{\left(1+\overline{\mathcal{M}}_{2}\right)}{\overline{\mathcal{M}}_{2}} B_{2}^{+} e^{j \omega\left(x-l_{1}\right) / \bar{c}_{2}\left(1+\bar{M}_{2}\right)}-\frac{\left(1-\overline{\mathcal{M}}_{2}\right)}{\overline{\mathcal{M}}_{2}} B_{2}^{-} e^{-j \omega\left(x-l_{1}\right) / \bar{c}_{2}\left(1-\bar{M}_{2}\right)} \\
{\left[\frac{\left(1+\overline{\mathcal{M}}_{1}\right)}{\zeta_{1}}+\frac{K\left(1+\overline{\mathcal{M}}_{1}\right)}{\eta_{p} \gamma \overline{\mathcal{M}}_{1}}\right] B_{1}^{+} e^{j \omega x / \bar{c}_{1}\left(1+\bar{M}_{1}\right)}+\left[\frac{\left(1-\overline{\mathcal{M}}_{1}\right)}{\zeta_{1}}-\frac{K\left(1-\overline{\mathcal{M}}_{1}\right)}{\eta_{p} \gamma \overline{\mathcal{M}}_{1}}\right] B_{1}^{-} e^{-j \omega x / \bar{c}_{1}\left(1-\bar{M}_{1}\right)}=} \\
\frac{\left(1+\overline{\mathcal{M}}_{2}\right)}{\zeta_{2}} B_{2}^{+} e^{j \omega\left(x-l_{1}\right) / \bar{c}_{2}\left(1+\bar{M}_{2}\right)}+\frac{\left(1-\overline{\mathcal{M}}_{2}\right)}{\zeta_{2}} B_{2}^{-} e^{-j \omega\left(x-l_{1}\right) / \bar{c}_{2}\left(1-\bar{M}_{2}\right)}
\end{gathered}
$$

The inlet acoustic condition (at $x=0$ ) is given by the inlet reflection coefficient as $R_{\text {in }}=$ $B_{1}^{+} / B_{1}^{-}$. Combining Eqs. (36) and (37) with the inlet acoustic condition and making the incoming perturbation to be $\hat{w}_{2}^{-}=1$ leads to a $4 \times 4$ system as follows: 


$$
\mathcal{C}\left(\begin{array}{c}
B_{1}^{+} \\
B_{1}^{-} \\
B_{2}^{+} \\
B_{2}^{-}
\end{array}\right)=\left(\begin{array}{l}
0 \\
0 \\
0 \\
1
\end{array}\right)
$$

where the matrix $\mathcal{C}$, for a compact compressor placed at $x=l_{1}$, is given by

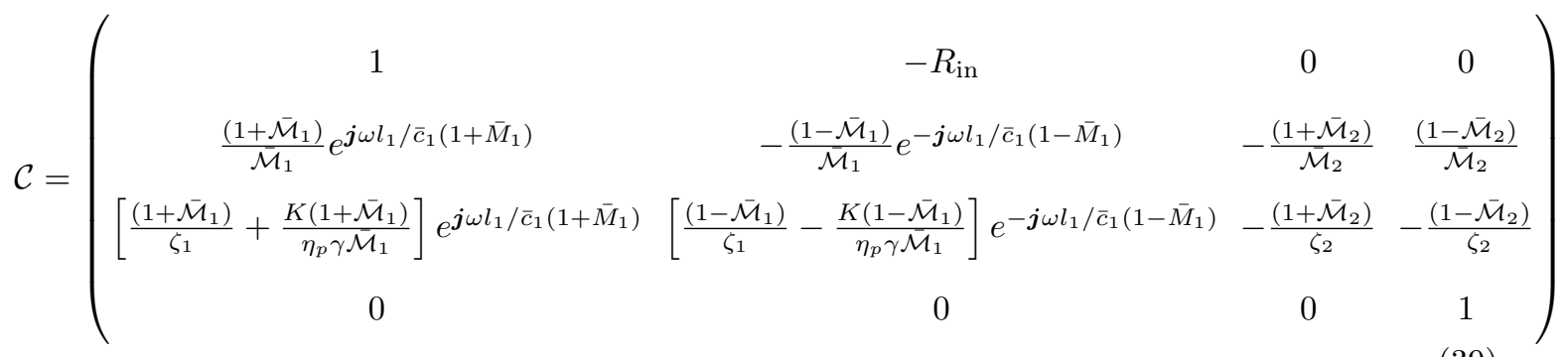

\section{B. Inlet acoustic condition}

The acoustic flux $f_{\text {ac }}$, normalized by the mean value of the energy flux, is defined as

$$
\frac{f_{\mathrm{ac}}}{\overline{\dot{m}} \bar{h}_{t}}=\frac{\dot{m}^{\prime}}{\overline{\dot{m}}} \frac{T_{t}^{\prime}}{\bar{T}_{t}}
$$

Replacing now the definition of $f_{\mathrm{ac}}$ by the left handside of Eqs. (22) and (23) and neglecting entropy waves $\left(\hat{w}^{S}=0\right)$ leads to a definition of the acoustic flux written in the form:

$$
\frac{f_{\text {inlet }, \text { ac }}}{\bar{m}_{1} \bar{h}_{1, t}}=\left[\frac{\left(1+\overline{\mathcal{M}}_{1}\right)}{2 \overline{\mathcal{M}}_{1}} \hat{w}_{1}^{+}-\frac{\left(1-\overline{\mathcal{M}}_{1}\right)}{2 \overline{\mathcal{M}}_{1}} \hat{w}_{1}^{-}\right]\left[\frac{\left(1+\overline{\mathcal{M}}_{1}\right)}{2 \zeta_{1}} \hat{w}_{1}^{+}+\frac{\left(1-\overline{\mathcal{M}}_{1}\right)}{2 \zeta_{1}} \hat{w}_{1}^{-}\right]
$$

In order to focus on the effect of the acoustic energy generated inside the system on the outlet reflection coefficient $R_{\text {out }}$, it is mandatory to assure that no acoustic flux is being imposed at the inlet of the configuration. For that purpose, one may assume either $\dot{m}^{\prime}=0$ or $\dot{T}_{t}^{\prime}=0$. For open apertures (not a wall), the second alternative is better suited. Satisfying $T_{1, t}^{\prime} / \bar{T}_{1, t}=0$ leads to a ratio between the downstream and upstream acoustic waves given by:

$$
R_{\text {in }}=\frac{w^{+}}{w^{-}}=B^{+} / B^{-} e^{j \phi}=\frac{\left(1-\overline{\mathcal{M}}_{1}\right)}{\left(1+\overline{\mathcal{M}}_{1}\right)} e^{j \pi}
$$


This reflection coefficient acts as a totally reflecting condition $\left(R_{\text {in }}=1 e^{j \pi}\right)$ for zero Mach number flows and tends to zero for inlet flows reaching a choked condition.

\section{Compressor modeling in Snozzle.}

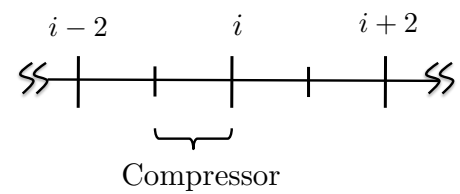

Fig. 7 Compact compressor in Snozzle

The compressor is modeled in Snozzle as an isentropic compact element, which is placed between the nodes $i-1$ and $i$ as illustrated in Fig. 7. Between these two nodes, the fluctuating mass and energy are related by:

$$
\begin{gathered}
\frac{\hat{\rho}_{i-1}}{\bar{\rho}_{i-1}}+\frac{\hat{u}_{i-1}}{\bar{u}_{i-1}}=\frac{\hat{\rho}_{i}}{\bar{\rho}_{i}}+\frac{\hat{u}_{i}}{\bar{u}_{i}} \\
\frac{1}{\zeta_{i-1}}\left(\overline{\mathcal{M}}_{i-1} \frac{\hat{u}_{i-1}}{\bar{c}_{i-1}}+\frac{\hat{\rho}_{i-1}}{\bar{\rho}_{i-1}}\right)+\frac{\gamma-1}{\eta_{p} \gamma} K\left(\frac{\hat{\rho}_{i-1}}{\bar{\rho}_{i-1}}+\frac{\hat{u}_{i-1}}{\bar{u}_{i-1}}\right)=\frac{1}{\zeta_{i}}\left(\overline{\mathcal{M}}_{i} \frac{\hat{u}_{i}}{\bar{c}_{i}}+\frac{\hat{\rho}_{i}}{\bar{\rho}_{i}}\right)
\end{gathered}
$$

where $\zeta=1+\frac{1}{2}(\gamma-1) \overline{\mathcal{M}}^{2}$ and $K$, defined in Eq. (34), is a constant related to the characteristics of the compressor and the mass flux at the operating point. The fluctuating velocity at the compressor inlet $\hat{u}_{i, 2}$ and the fluctuating density at the compressor outlet $\hat{\rho}_{i+1,1}$ are expressed as function of neighboring parameters:

$$
\begin{gathered}
\hat{u}_{\text {ups }}=\hat{u}_{i-1}=\Gamma \cdot \hat{u}_{i}+\Lambda \cdot \hat{\rho}_{i-1} \\
\hat{\rho}_{\text {down }}=\hat{\rho}_{i}=\Upsilon \cdot \hat{u}_{i}+\Omega \cdot \hat{\rho}_{i-1}
\end{gathered}
$$

After combining Eqs. (43) and (44) with Eqs. (45) and (46), it can be found that the constants $\Gamma, \Lambda, \Upsilon$ and $\Omega$ are defined by:

$$
\Gamma=\frac{\bar{u}_{i-1} \bar{c}_{i-1}^{2} \zeta_{i-1} \eta_{p} \gamma\left(\bar{u}_{i}^{2}-\bar{c}_{i}^{2}\right)}{\bar{u}_{i} \bar{c}_{i}^{2} \Theta}
$$




\begin{tabular}{|c||c|c|c|}
\hline Cases Studied & $\bar{\pi}_{c}=1.0$ & $\bar{\pi}_{c}=2.0$ & $\bar{\pi}_{c}=5.0$ \\
\hline \hline$\hat{\pi}_{c}=0$ & A01 & A02 & A03 \\
\hline$\hat{\pi}_{c}=-1.0$ & & A04 & \\
\hline$\hat{\pi}_{c}=-1.5$ & & A05 & \\
\hline
\end{tabular}

Table 3 Five different configurations as function of $\bar{\pi}_{c}$ and $\hat{\pi}_{c}$. For this cases the polytropic efficiency $\eta_{p}$ is considered equal to one. Values of $\hat{\pi}_{c}$ and $\bar{\pi}_{c}$ are taken from typical values of actual aeronautical compressors.

$$
\begin{gathered}
\Lambda=\frac{\bar{u}_{i-1} \bar{c}_{i-1}^{2}\left[\eta_{p} \gamma\left(\zeta_{i-1}-\zeta_{i}\right)-\zeta_{i} \zeta_{i-1} K(\gamma-1)\right]}{\bar{\rho}_{i-1} \Theta} \\
\Upsilon=\frac{\bar{\rho}_{i}\left[\bar{u}_{i}^{2} \bar{c}_{i-1}^{2} \zeta_{i-1} \eta_{p} \gamma-\bar{u}_{i-1}^{2} \bar{c}_{i}^{2} \zeta_{i} \eta_{p} \gamma-\bar{c}_{i}^{2} \bar{c}_{i-1}^{2} \zeta_{i} \zeta_{i-1} K(\gamma-1)\right]}{\bar{c}_{i}^{2} \bar{u}_{i} \Theta} \\
\Omega=\frac{\bar{\rho}_{i}\left[\zeta_{i} \eta_{p} \gamma\left(\bar{u}_{i-1}^{2}-\bar{c}_{i-1}^{2}\right)-\bar{c}_{i-1}^{2} K(\gamma-1)\left(\zeta_{i}^{2}-\zeta_{i} \zeta_{i-1}\right)\right]}{\bar{\rho}_{i-1} \Theta}
\end{gathered}
$$

where

$$
\Theta=\bar{u}_{i-1}^{2} \zeta_{i} \eta_{p} \gamma+\bar{c}_{i-1}^{2} \zeta_{i} \zeta_{i-1} K(\gamma-1)-\bar{c}_{i-1}^{2} \zeta_{i-1} \eta_{p} \gamma
$$

These parameters are injected into the discretized equations of continuity Eq. (11) and momentum Eq. (12) at nodes $i-1$ and $i$. In addition to validate the numerical tool Snozzle by comparing its results to the analytical ones, this section aims at studying the effect of a compact compressor on the outlet reflection coefficient defined as $R_{\text {out }}=\hat{w}_{2}^{+} / \hat{w}_{2}^{-}$of a constant section intake. Five cases are evaluated as shown in table 3 and Fig. 8 . No $\hat{\pi}_{c}>0$ is considered here as it corresponds to unstable operating points. For the cases shown in table 3, the downstream mean values of temperature, pressure and Mach number are $\bar{T}_{2}=600 \mathrm{~K}, \bar{p}_{2}=200 \mathrm{kPa}$ and $\overline{\mathcal{M}}_{2}=0.1$, respectively.

\section{Results}

Figures 9 and 10 show the different values of the reflection coefficient $R_{\text {out }}=\hat{w}_{2}^{+} / \hat{w}_{2}^{-}$for the cases shown in table 3. This reflection coefficient is plotted against the non-dimensional frequency 


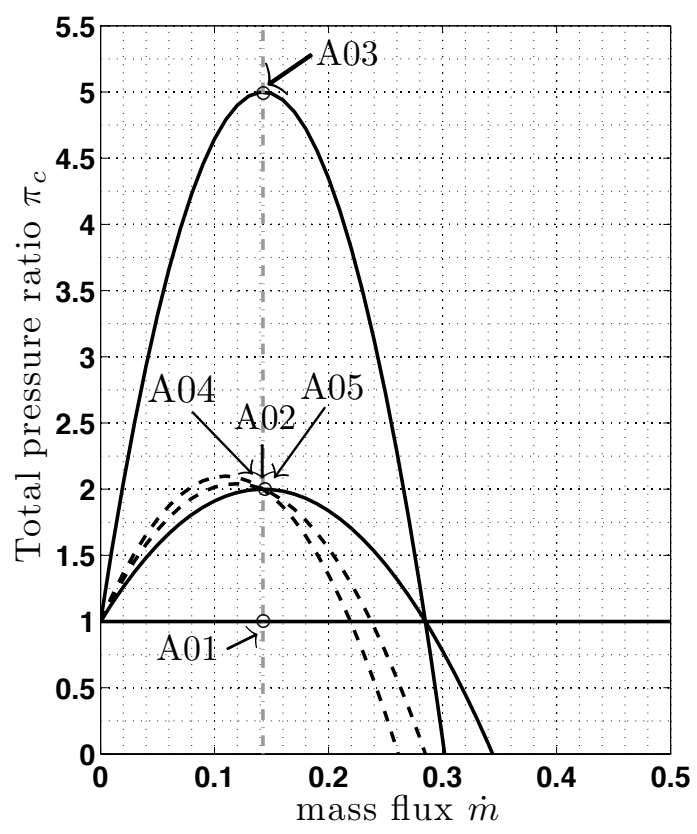

Fig. 8 Cases evaluated (see table 3). They are given by the intersection between the polynomial curves and the vertical line at $\dot{m}=0.1425 \mathrm{~kg} / \mathrm{s}$. Dashed lines corresponds to the cases for which the slope of $\pi_{c}$ is $\hat{\pi}_{c}=-1.0(\mathbf{A 0 4})$ and $\hat{\pi}_{c}=-1.5(\mathbf{A 0 5})$.

$\Omega=f l_{n} / \bar{c}_{2}$. As a first observation it is seen that numerical results coming from the Helmholtz solver match the analytical results very well for all cases. On the one hand, the modulus of $R_{\text {out }}$ seems to be a quantity that does not depend on the mean value of the pressure ratio at all: $|R|_{\text {out }}=0.81$ for all cases shown in Fig. 9. This result is actually expected since Eq. (35) tells us that the normalized fluctuation energy $\hat{T}_{t} / \bar{T}_{t}$ must remain constant through the compact compressor as long as there is no pressure ratio fluctuations $\left(\hat{\pi}_{c}=0\right.$ or $\left.K=0\right)$. Figure 9 also shows, on the other hand, that the argument of $R_{\text {out }}$ strongly depends on the value of $\bar{\pi}_{c}$. In a reference case $\left(\bar{\pi}_{c}=1\right)$, the argument of $R_{\text {out }}$ evolves linearly as expected for an homogeneous mean field. However, as soon as $\bar{\pi}_{c}$ increases, the argument of $R_{\text {out }}$ moves away from this linear behavior and takes values that can be actually phase shifted up to $\pi$ with respect to the reference case. It will be observed in the next section that small changes in the argument of $R$ for an intake outlet can lead to very different estimations of the acoustic modes of a given combustion chamber.

In the low frequency limit, the downstream acoustic wave $\hat{w}_{2}^{+}$can be analytically described by 

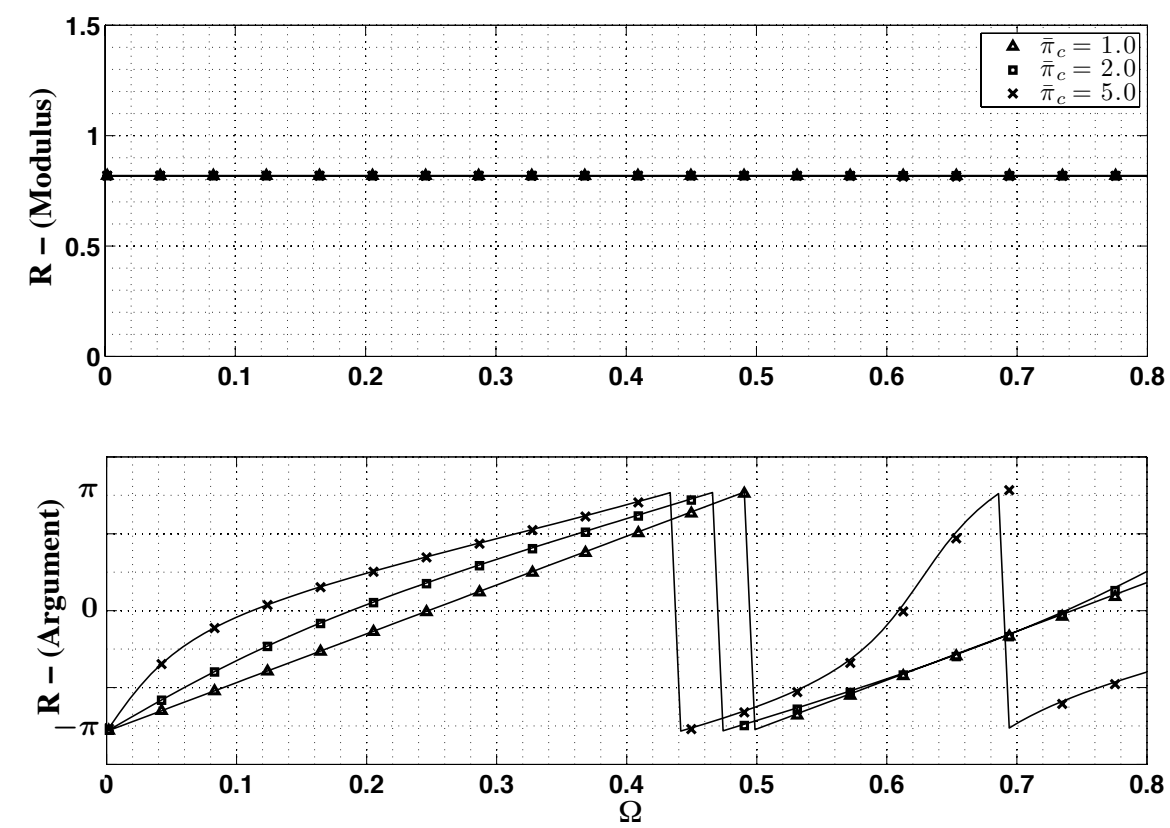

Fig. 9 Reflection coefficient at intake's outlet. The fluctuating value $\pi_{c}^{\prime}$ is set to zero while the mean value $\bar{\pi}_{c}$ varies from 1.0 until 5.0. Symbols and straight lines represent numerical and analytical results respectively.

the following relation:

$$
\hat{w}_{2}^{+}=\frac{\left(1-\overline{\mathcal{M}}_{2}\right)\left(K \zeta_{2}+\gamma \overline{\mathcal{M}}_{2}\right)}{\left(1+\overline{\mathcal{M}}_{2}\right)\left(K \zeta_{2}-\gamma \overline{\mathcal{M}}_{2}\right)} \hat{w}_{2}^{-}
$$

According to Eq. (52), values of $\hat{w}_{2}^{+}$, and therefore $R_{\text {out }}$, can be strongly amplified when $K \rightarrow$ $\gamma \overline{\mathcal{M}}_{2} / \zeta_{2}$. Compressor operating point is most of the time established for cases in which $\hat{\pi}_{c}<0$ (see Fig. 6 ). For these cases the definition of the downstream acoustic wave $\hat{w}_{2}^{+}$(Eq. (52)) does not present any singularity since $K$ is always negative. Attention is only paid then to $\hat{\pi}_{c} \leq 0$. Fig. 10 shows values of $\hat{\pi}_{c}$ going from 0 until -1.5. On the one hand, it is observed from Fig. 10 that the values of $R_{\text {out }}$, for the modulus, are always below those for which $\hat{\pi}_{c}=0$. On the other hand, values for the argument of $R_{\text {out }}$ vary very little when decreasing the fluctuating quantity $\hat{\pi}_{c}$.

\section{E. Model extension to a more general compressor}

In the present work the whole compressor is modeled as a compact element that adds energy to the system by modifying its total pressure and kinetic energy. This can be extended to a non 

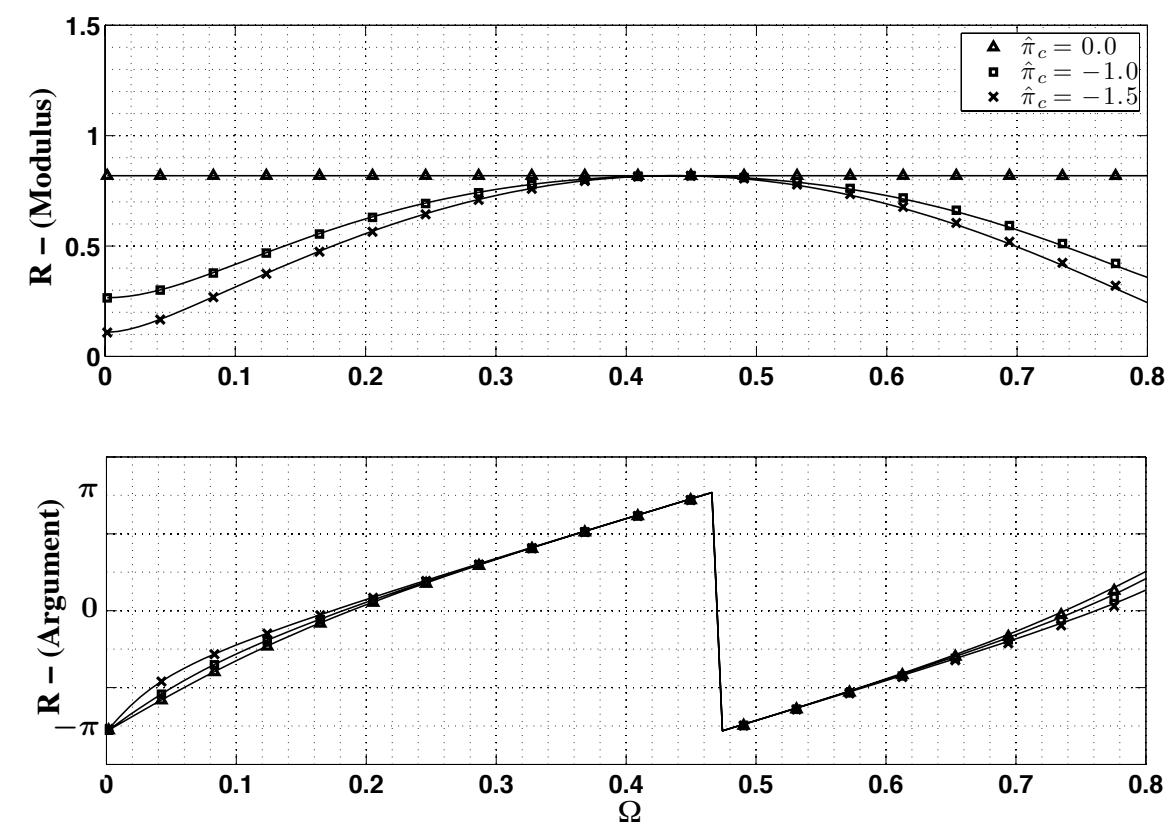

Fig. 10 Reflection coefficient at intake's outlet. The mean value of the pressure ratio remains constant at $\bar{\pi}_{c}=2.0$ while the fluctuating value $\hat{\pi}_{c}$ varies from 0.0 until $\mathbf{- 1 . 5}$. Symbols and straight lines represent numerical and analytical results respectively.

compressor by considering a sum of rotor/stator combinations. On the one hand, each rotor row could be modeled as a compact element that adds total enthalpy to the system. On the other hand, each stator row could be modeled as an isentropic element that adds a phase lag to the incoming acoustic waves. Finally, the sum of the contributions of each individual element would characterize the acoustics of an entire compressor stage. This a convenient approach in the framework of linear acoustics.

\section{Computation of the Reflection Coefficient on the Inlet air circuit of an Helicopter}

\section{combustor chamber}

The numerical tool described above can be used to compute the equivalent impedance of any air-intake duct with or without total enthalpy change at the compressor location. It is well known that the resonant modes of any combustion chamber are strongly dependent on the inlet and outlet acoustic boundary conditions used [13]. On the one hand, the flow at the outlet of the combustion chamber (the high pressure distributor) is generally choked [36]. In this case, and for low frequencies, it is known that the reflection coefficient can be considered close to that of a rigid wall. On the 
other hand, the same cannot be stated at the inlet, as acoustic properties of the combustion chamber inlet are fully dependent on the upstream configuration of the combustor. Wrong predictions of the resonant frequencies can be caused by simplified treatment of the inlet impedance.

Aeronautical engines are complex systems in which the combustion chamber is present just in a small region after the compressor stages. From the point of view of the combustor, the upstream region is then composed by several compression stages in which acoustics depends on enthalpy jumps (compressors), the intake inlet acoustic condition (usually related to the atmosphere) and the geometry of the entire intake system. In the remaining of this paper, the numerical tool discussed in sections II-V is used to assess the acoustic reflection coefficient at the inlet of the combustion chamber, i.e outlet of the air-intake duct $R_{\text {out }}$. The intake belongs to an helicopter combustor and is shown in Fig. 11. Once this acoustic boundary condition is evaluated, it can be imposed in a Helmholtz solver computation or in a Large Eddy Simulation [21, 38]

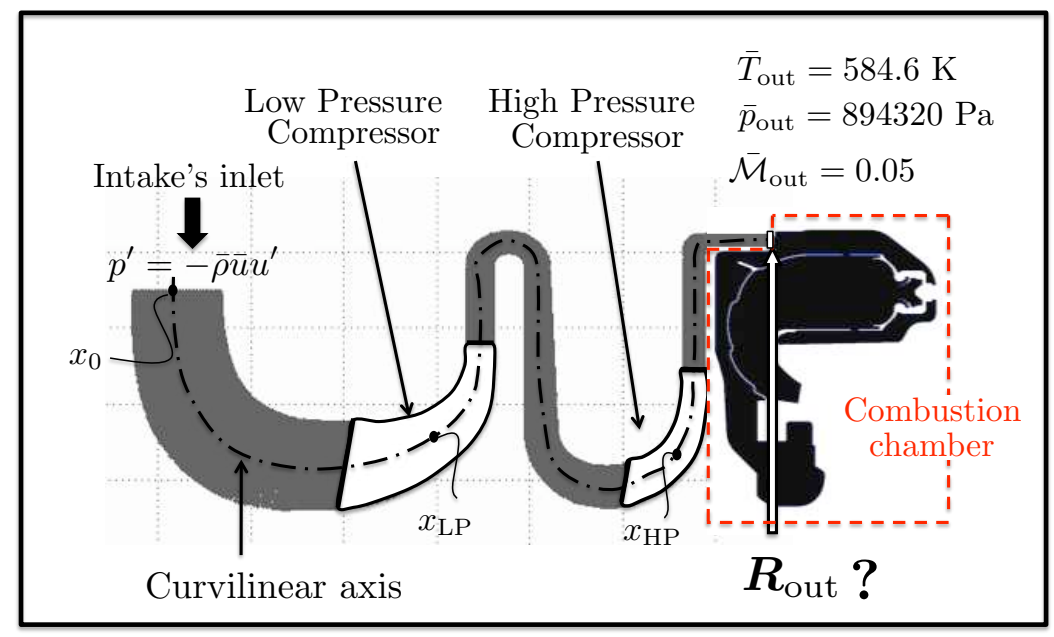

Fig. 11 Air-intake duct configuration.

\section{A. Mean parameters of the Intake Duct}

SNozzle requires the mean temperature and pressure, the mean value of the Mach number at the outlet and the section area at several points of the curvilinear axis of the air-intake duct. The position and the total pressure jump of the compressors is also needed in order to characterize the mean flow. It is assummed, when describing the mean flow along the intake duct, that the 
compressors are acoustically compact. It means that the curvilinear length of the compressors (see Figs. 11 and 14) is small compared to the acoustic wavelengths of interest. For this reason, the evolution of the mean flow along the compressors is left aside and, instead, a sudden jump in the total mean flow quantities is considered at the middle of the curvilinear axis of each compressor (dashed lines in Fig. 11).

In the present helicopter system, the compression of gases is not performed in an isentropic manner: instead of one, the polytropic coefficient has a value around $\eta_{p}=0.88$ for both compressor stages. This value of $\eta_{p}$ will be accounted for in one of the three cases considered for the evaluation of the mean field. This is done in order to illustrate the effect of small changes in the mean field on the estimation of the reflection coefficient. It should be pointed out that although the compressors are not anymore considered isentropic for the mean field, they are still taken as isentropic for the fluctuating field since no entropy waves are assumed to be generated (and therefore $\eta_{p}=1$ in Eq. (44)). Figure 12 shows the mean field of total pressure and total temperature corresponding to the helicopter intake considered for three different cases: a) constant total pressure along the intake duct, b) isentropic mean flow with isentropic total pressure jumps due to compressors and c) isentropic mean flow with non-isentropic total pressure jumps due to actual compressors with $\eta_{p}<1$.

It should be noted that only by modeling the total enthalpy jumps through the compressors it is possible to estimate a reliable mean field, notably the Mach number that in fact determines the acoustic wavelengths. Most of existing quasi 1D -LEE solvers, however, do not account for any change in total enthalpy [25, 27]. An illustration of the Mach number (normalized by the Mach number at the outlet of the intake) for the three cases already mentioned is shown in Fig. 13. As expected the introduction of the two compressor rows induces a strong variation of Mach number. Accounting for the polytropic efficiency only reduces this parameter slightly mainly in the low pressure compressor. 


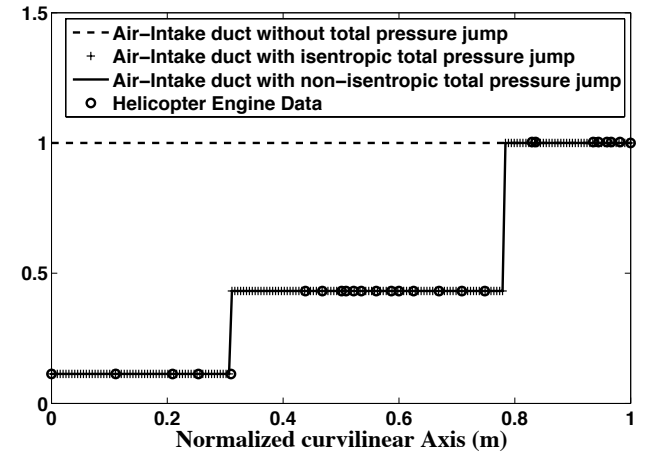

a) Normalized total pressure

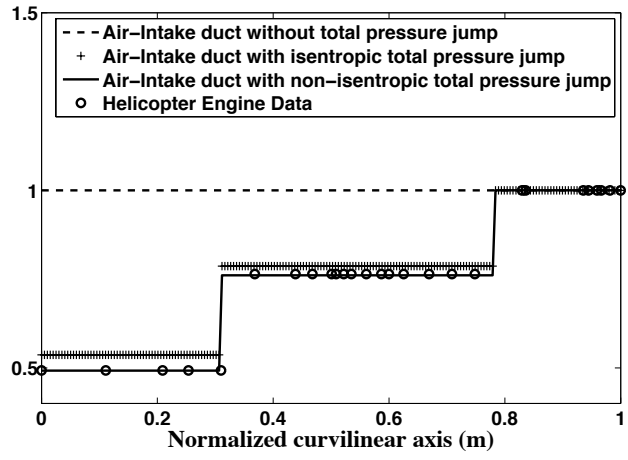

b) normalized total temperature

Fig. 12 Total pressure and total temperature profiles normalized by the corresponding values at the outlet of the air-intake duct.

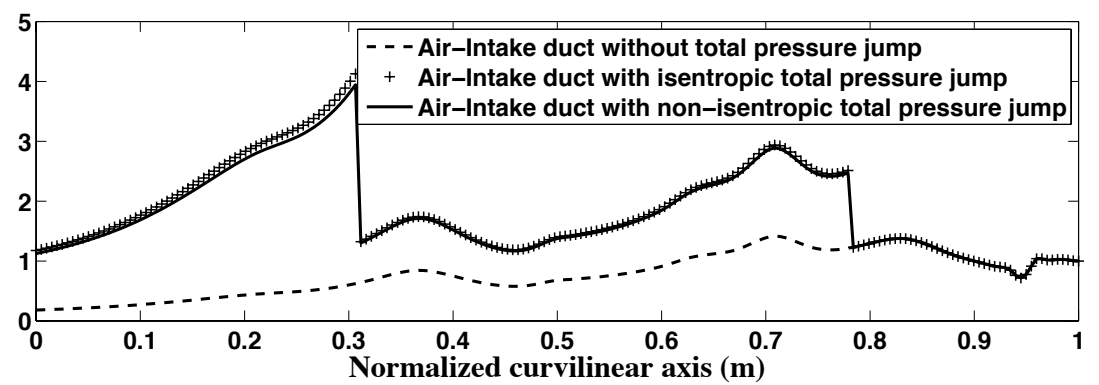

Fig. 13 Mach number normalized by the corresponding values at the outlet of the air-intake duct.

\section{B. Results}

As described in section $\mathrm{V}$, the reflection coefficient at the outlet of the intake duct $R_{\text {out }}$ is estimated by evaluating the ratio between the reflected acoustic wave $\hat{w}_{2}^{+}$and the imposed acoustic wave $\hat{w}_{2}^{-}$as illustrated in Fig. 14. The acoustic reflection at the inlet of the air-intake $R_{\text {in }}$ is partially reflecting and is associated to Eq. (42), as done in section V for the ideal 1D intake. The two compressors present in the inlet-air duct are assumed acoustically compact and the associated total pressure ratio $\pi_{c}$, which is direclty related to the mass flow by the characteristic curves (see Fig. 6), is given by Eq. (34). From section V it was observed, for an ideal $1 \mathrm{D}$ intake, that no significant changes in the estimation of $R_{\text {out }}$ (mainly phase) are obtained when either considering or not the fluctuating contribution $\hat{\pi}_{c}$. Therefore, in this study only the mean contribution of the total pressure ratio $\bar{\pi}_{c}$ of each compressor is accounted for. 


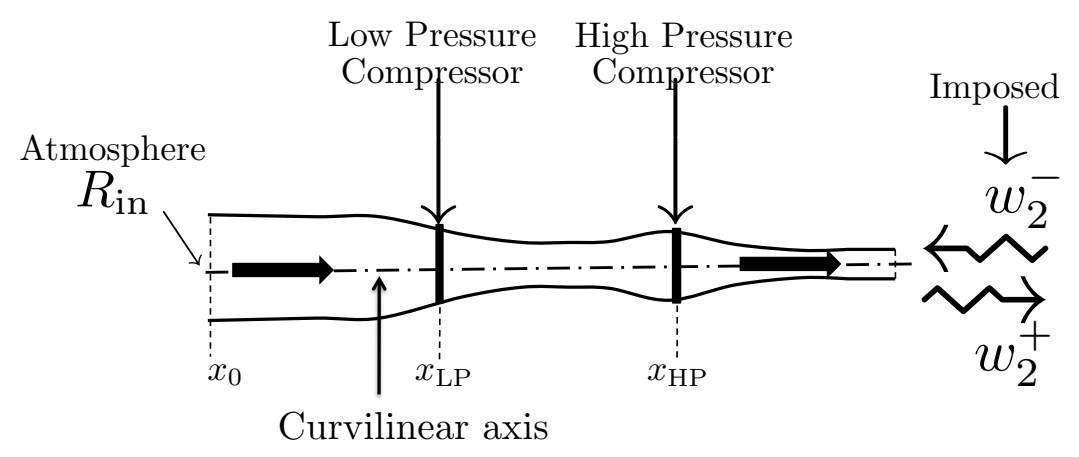

Fig. 14 Model of air-intake duct.

Figure 15 shows both the estimated modulus and argument of $R_{\text {out }}$ for the above three cases studied. These two parameters are function of the frequency that ranges from 0 to $600 \mathrm{~Hz}$. This upper value is taken assuming that a compressor remains acoustically compact if its length is ten times smaller than the acoustic wave length. The module of $R_{\text {out }}$ has a constant value of 0.9 when no total pressure ratio is considered whereas it smoothly oscillates between 0.9 and 0.98 when accounting for compact compressors. In spite of this, it can be considered that there is not any significative difference between the three cases studied for values of the module of $R_{\text {out }}$. On the contrary big changes are found, as expected from the results of the previous section, when observing the values of the argument of $R_{\text {out }}$ : the phase difference between $\hat{w}_{2}^{+}$and $\hat{w}_{2}^{-}$increases faster as the frequency grows for the two cases accounting for total pressure changes. This fact may influence the resonance frequency of the combustion chamber dramatically. The influence of considering a polytropic coefficient when computing the mean field is finally observed. It is seen in Fig. 15 that almost no differences exist for both modulus and argument of $R_{\text {out }}$ if the mean field of the temperature is modified by applying this value of $\eta_{\mathrm{pol}}$.

\section{Helmholtz Solver Computation}

AVSP $[13,20,21,39]$ is the Helmholtz solver used here to compute the different acoustic modes of the aero-engine of interest. Most of the time the outlet of the combustion system is choked, which corresponds, for low frequencies, to an acoustic condition similar as $u^{\prime}=0$. The acoustic condition at the inlet is most of the time unknown. Usually, this boundary condition is also approximated by 

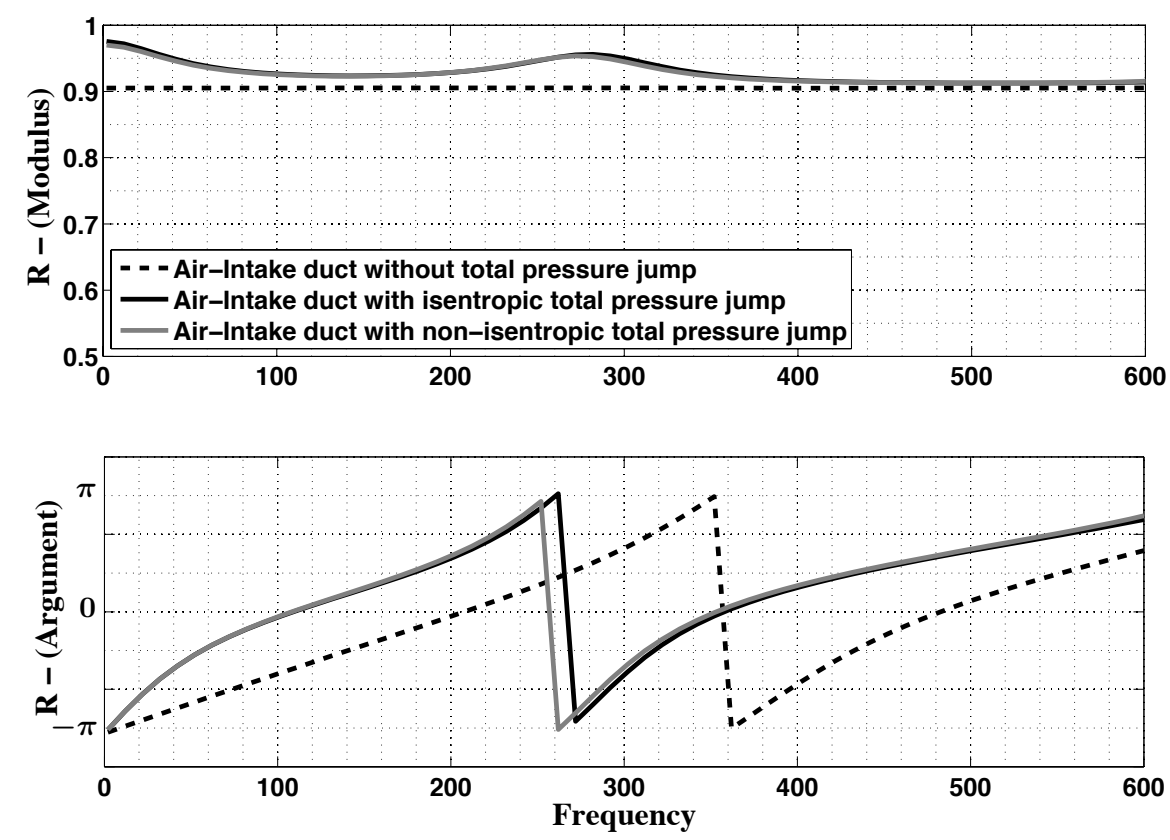

Fig. 15 Reflection Coefficient at the outlet of the helicopter intake duct.

$u^{\prime}=0$ even though it is actually not correct. The purpose of this section is then to show how the first two acoustic modes of the chamber under study vary according to the imposed inlet acoustic condition $R_{\text {out }}{ }^{[\dagger]}$.

Figure 16 shows the values of the resonance frequency as function of the modulus and argument of $R_{\text {out }}$. First, it is assumed an inlet reflection coefficient in which $\arg \left(R_{\text {out }}\right)=0$ and $\left|R_{\text {out }}\right|$ varies from 0.1 to 10 . This large range is studied to gain an overview of the associated effects on the combustion chamber acoustics. Clearly, large values of $\left|R_{\text {out }}\right|$ would mean that external acoustic energy enters the system. Second, $\left|R_{\text {out }}\right|$ is maintained equal to 0.9 and $\arg \left(R_{\text {out }}\right)$ is varied from $-\pi$ to $\pi$. It is seen that a significant variation is produced on the resonant frequency of the first two acoustic modes when the phase of $R_{\text {out }}$ is changed at the inlet of the combustor. This is notably the case for the first mode since this frequency can vary up to 400 when modifying the phase from $-\pi$ to $\pi$. The same shift of phase can modify the resonant frequency of the second mode until 100 Hz. These results are obtained maintaining $\left|R_{\text {out }}\right|=0.9$. Similar results are obtained for a fixed modulus $\left|R_{\text {out }}\right|=1.0$, and therefore it can be stated that results shown in Fig. 16 are representative

[†] In this section $R_{\text {out }}$ is associated with the inlet of the combustion chamber, i.e. the outlet of the air-intake duct (see Fig. 11 and Fig. 18). 


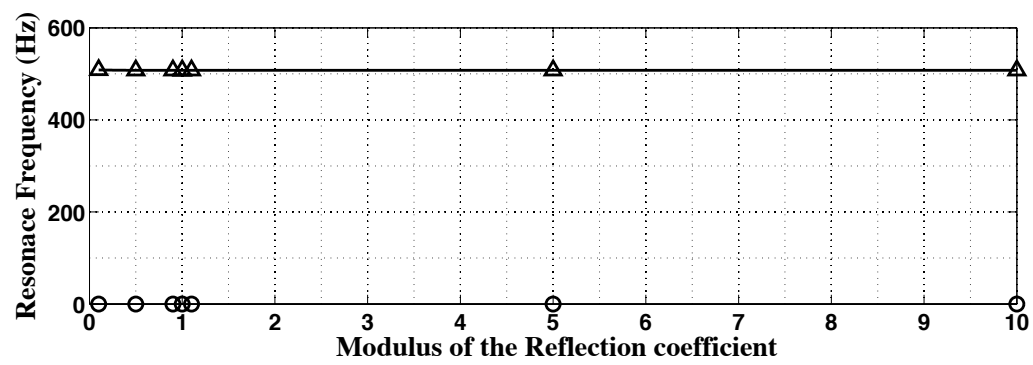

(a) Resonance frequency vs. $|R|$ - for $\operatorname{Arg}(\mathrm{R})=0$

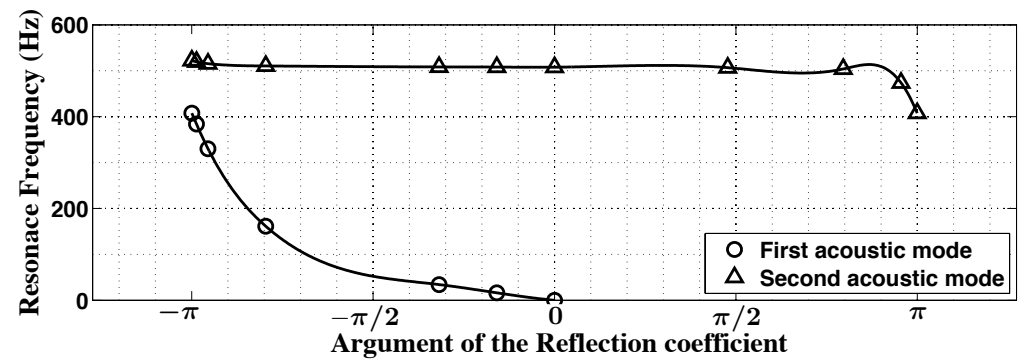

(b) Resonance frequency vs. $\operatorname{Arg}(\mathrm{R})$ - for $|R|=0.9$

Fig. 16 Dependance on the inlet reflection coefficient $\left(R_{\text {out }}\right.$ of the Air-intake) of the combustion chamber resonance frequencies of the first two acoustic modes - Helmholtz solver results.

of the range of $\left|R_{\text {out }}\right|$ of interest (Fig. 15).

Varying the modulus of the reflection coefficient $R_{\text {out }}$ does not imply a significative change in any of the first two acoustic modes of the combustor. These results are expected, since only the phase of $R_{\text {out }}$ can notably alter the wavelength of the acoustic fluctuations inside the system, and, therefore, the corresponding resonance frequency. The modulus, on the contrary, does not alter considerably this wave length but, instead, acts on the amount of acoustic energy subtracted from the system. This should be clear if the modulus and argument of $R_{\text {out }}$ are thought as the acoustic resistance (real part of the acoustic impedance) and acoustic reactance (imaginary part of the acoustic impedance) of the acoustic boundary, respectively.

Results shown in Fig. 16 are obtained for a long range of values of $R_{\text {out }}$ by changing either the associated module or phase. Now, the reflection coefficient $R_{\text {out }}$ obtained by the procedure of section VI is used to compute the acoustic modes of the combustion chamber. The effect of compressors at the air-intake duct can then be assessed.

With this in mind, and in order to get an overall view of the possible resonant frequencies of the 


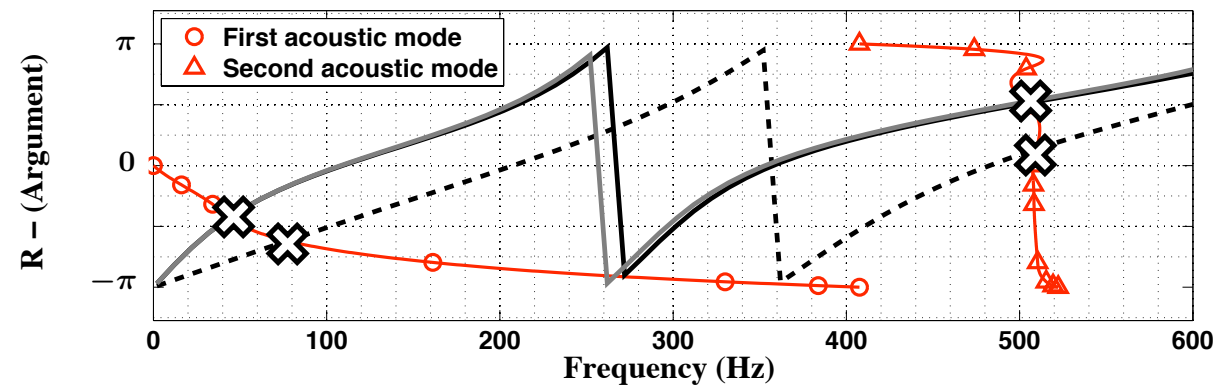

Fig. 17 Reflection coefficient $R_{\text {out }}$ at the outlet of the helicopter intake duct (grey lines). Resonance frequency of the combustion chamber when varying the argument of the reflection coefficient at its inlet (red lines). Intersection of red and grey lines (marked with crosses) represent expected resonance frequencies of the combustion chamber when coupled with the air intake under study.

combustion chamber, Fig. 15 and Fig. 16(b) are merged and represented in Fig. 17. Note that the axes of Fig. 16(b) are inverted in Fig. 17. The points of intersection between the red and grey lines, marked with a cross in Fig. 17, may represent the resonance frequency of the combustion chamber when coupled with the air intake under study. The frequency associated with the second acoustic mode is practically not altered if $R_{\text {out }}$ corresponds to an intake either with or without compressors: in both cases it has a value around $500 \mathrm{~Hz}$. In contrast, the frequency corresponding to the first acoustic mode is clearly influenced by the way $R_{\text {out }}$ is evaluated. Whereas it has values of $\approx 40 \mathrm{~Hz}$ if compressors are included in the computation of $R_{\text {out }}$, it presents values of $\approx 80 \mathrm{~Hz}$ if compressors are not included in the computation. In order to verify this graphical analysis, computations are carried out with the Helmholtz solver. Results show that, on the one hand, when compressors are considered to estimate $R_{\text {out }}$, the resonant frequencies are $51.5 \mathrm{~Hz}$ and $507.4 \mathrm{~Hz}$ for the first and second acoustic mode, respectively. The second acoustic mode is illustrated in Fig. 18. On the other hand, when compressors are not accounted for in the air-intake, the combustion chamber resonant frequencies are $86.8 \mathrm{~Hz}$ and $506.6 \mathrm{~Hz}$, for the first and second acoustic mode respectivelty. It is then observed that the value of $R_{\text {out }}$ used to estimate the first acoustic mode of the combustion chamber is not trivial. The approach described in the present study might be useful in order to reach an accurate prediction of this frequency of oscillation. 


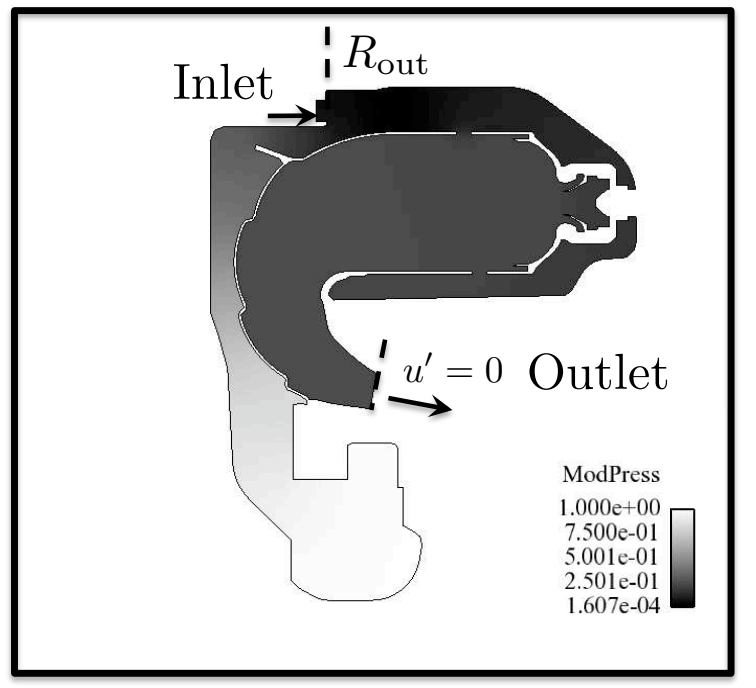

(a) $|\hat{p}|$

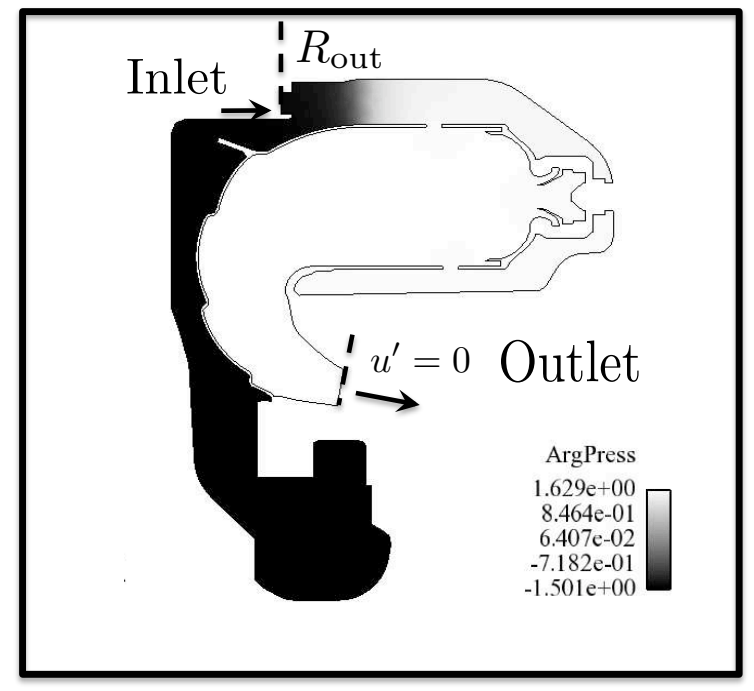

(b) $\operatorname{Arg}(\hat{p})$

Fig. 18 Second acoustic mode of the Aeroengine combustor $(507.4 \mathrm{~Hz})$. $R_{\text {out }}$ for an air-intake duct with compressors.

\section{Conclusions}

A numerical tool has been developed to compute the acoustic field of quasi-1D configurations accounting for total enthalpy jumps. This kind of situation is in fact present in the inlet-air circuit of actual aeronautical engines. Modeling this total pressure jump is not an easy task in the framework of a quasi 1D system: the concept of rothalpy cannot be developed. A model is then considered in which the total pressure ratio $\pi_{c}$ of each compressor is related to the characteristics curves of this element at a given operating point. In this way, it is possible to express the mean and fluctuating values of $\pi_{c}$ as a function of the mass flow and, as a consequence, to formulate two conservation equations through this interface. Analytical solutions are subsequently developed for compact compressors immersed in constant section ducts. These solutions are compared to the results obtained by the Helmholtz solver for validation purposes. Finally, the outlet reflection coefficient $R_{\text {out }}$ of an air-inlet circuit of an actual combustion chamber is computed. Significative differences on the estimate of $R_{\text {out }}$ results when comparing computations in which total pressure jumps are either considered or not. It has been also observed that the argument of $R_{\text {out }}$, on the contrary to the modulus of $R_{\text {out }}$, plays an important role on the value of the eigenfrequencies of the combustion chamber studied. 


\section{Appendix}

In order to validate SNozzle, analytical solutions are derived for compact regions where the mean enthalpy or entropy may change. The starting point for this derivation are the 1D Euler equations for mass, momentum and total enthalpy.

$$
\begin{aligned}
\frac{\partial \rho}{\partial t}+\frac{\partial}{\partial x}(\rho u) & =0 \\
\frac{\partial \rho u}{\partial t}+\frac{\partial}{\partial x}\left(\rho u^{2}\right) & =-\frac{\partial p}{\partial x}+\mathcal{F} \\
\frac{\partial \rho h_{t}}{\partial t}+\frac{\partial}{\partial x}\left(\rho u h_{t}\right)-\frac{\partial p}{\partial t} & =\dot{\mathcal{Q}}+\mathcal{W}
\end{aligned}
$$

Integrating over the $1 \mathrm{D}$ domain between positions $x_{1}$ and $x_{2}$ results in

$$
\begin{gathered}
\int_{x_{1}}^{x_{2}} \frac{\partial \rho}{\partial t} d x+\int_{x_{1}}^{x_{2}} \frac{\partial}{\partial x}(\rho u) d x=0 \\
\int_{x_{1}}^{x_{2}} \frac{\partial \rho}{\partial t} d x+\left.(\rho u)\right|_{x_{1}} ^{x_{2}}=0 \\
\int_{x_{1}}^{x_{2}} \frac{\partial \rho u}{\partial t} d x+\int_{x_{1}}^{x_{2}} \frac{\partial}{\partial x}\left(\rho u^{2}\right) d x+\int_{x_{1}}^{x_{2}} \frac{\partial p}{\partial x} d x-\int_{x_{1}}^{x_{2}} \mathcal{F} d x=0 \\
\int_{x_{1}}^{x_{2}} \frac{\partial \rho u}{\partial t} d x+\left.\left(\rho u^{2}\right)\right|_{x_{1}} ^{x_{2}}+\left.p\right|_{x_{1}} ^{x_{2}}-\mathbb{F}=0 \\
\int_{x_{1}}^{x_{2}} \frac{\partial p}{\partial t} d x+\int_{x_{1}}^{x_{2}} \frac{\partial}{\partial x}\left(\rho u h_{t}\right) d x-\int_{x_{1}}^{x_{2}} \dot{\mathcal{Q}} d x-\int_{x_{1}}^{x_{2}} \mathcal{W} d x=0 \\
\int_{x_{1}}^{x_{2}} \frac{\partial p}{\partial t} d x+\left.\left(\rho u h_{t}\right)\right|_{x_{1}} ^{x_{2}}-\dot{\mathbb{Q}}-\mathbb{W}=0
\end{gathered}
$$

where the following notations have been introduced: $\int \dot{\mathcal{Q}} d x=\dot{\mathbb{Q}}, \int \mathcal{F} d x=\mathbb{F}$ and $\int \mathcal{W} d x=\mathbb{W}$. After linearizing and considering harmonic oscillations $\left(\phi^{\prime}=\hat{\phi} e^{-j \omega t}\right)$, Eqs. (56), (57) and (58) become

$$
\begin{gathered}
\left.\bar{\rho} \bar{u}\left(\frac{\hat{\rho}}{\bar{\rho}}+\frac{\hat{u}}{\bar{u}}\right)\right|_{x_{1}} ^{x_{2}}=j \omega \int_{x_{1}}^{x_{2}} \hat{\rho} d x \\
\left.\bar{\rho} \bar{u}^{2}\left(\frac{\hat{\rho}}{\bar{\rho}}+2 \frac{\hat{u}}{\bar{u}}\right)\right|_{x_{1}} ^{x_{2}}+\left.\bar{p}\left(\frac{\hat{p}}{\bar{p}}\right)\right|_{x_{1}} ^{x_{2}}-\hat{\mathbb{F}}=j \omega \int_{x_{1}}^{x_{2}}(\bar{\rho} \hat{u}+\hat{\rho} \bar{u}) d x \\
\left.\bar{\rho} \bar{u} \bar{h}_{t}\left(\frac{\hat{\rho}}{\bar{\rho}}+\frac{\hat{u}}{\bar{u}}+\frac{\hat{h}_{t}}{\bar{h}_{t}}\right)\right|_{x_{1}} ^{x_{2}}-\hat{\mathbb{Q}}-\hat{\mathbb{W}}=j \omega \int_{x_{1}}^{x_{2}}\left(\bar{\rho} \hat{h}_{t}+\hat{\rho} \bar{h}_{t}-\hat{p}\right) d x
\end{gathered}
$$


The contribution of the RHS terms on the above equations goes to zero for a compact region $\left(x_{1} \rightarrow x_{2}\right)$ as long as only bounded fluctuations exist within this region, as also assumed in the work of Dowling [4]. Equations (59), (60) and (61) are then simplified to

$$
\begin{gathered}
\frac{\hat{\rho}_{1}}{\bar{\rho}_{1}}+\frac{\hat{u}_{1}}{\bar{u}_{1}}=\frac{\hat{\rho}_{2}}{\bar{\rho}_{2}}+\frac{\hat{u}_{2}}{\bar{u}_{2}} \\
\bar{\rho}_{1} \bar{u}_{1}^{2}\left(\frac{\hat{\rho}_{1}}{\bar{\rho}_{1}}+2 \frac{\hat{u}_{1}}{\bar{u}_{1}}\right)+\bar{p}_{1}\left(\frac{\hat{p}_{1}}{\bar{p}_{1}}\right)+\hat{\mathbb{F}}=\bar{\rho}_{2} \bar{u}_{2}^{2}\left(\frac{\hat{\rho}_{2}}{\bar{\rho}_{2}}+2 \frac{\hat{u}_{2}}{\bar{u}_{2}}\right)+\bar{p}_{2}\left(\frac{\hat{p}_{2}}{\bar{p}_{2}}\right) \\
\bar{\rho}_{1} \bar{u}_{1} \bar{h}_{t 1}\left(\frac{\hat{\rho}_{1}}{\bar{\rho}_{1}}+\frac{\hat{u}_{1}}{\bar{u}_{1}}+\frac{\hat{h}_{t 1}}{\bar{h}_{t 1}}\right)+\hat{\mathbb{Q}}+\hat{\mathbb{W}}=\bar{\rho}_{2} \bar{u}_{2} \bar{h}_{t 2}\left(\frac{\hat{\rho}_{2}}{\bar{\rho}_{2}}+\frac{\hat{u}_{2}}{\bar{u}_{2}}+\frac{\hat{h}_{t 2}}{\bar{h}_{t 2}}\right)
\end{gathered}
$$

where the indices 1 and 2 denote the regions upstream or downstream of any infinitely thin compact element; $\hat{\mathbb{Q}}, \hat{\mathbb{F}}$ and $\hat{\mathbb{W}}$ being the total heat, force and work associated to this element.

\section{References}

[1] L. Rayleigh. The explanation of certain acoustic phenomena. Nature, July 18:319-321, 1878.

[2] T. Poinsot and S. Candel. Interactions between acoustics and combustion. In Acoustics 88., 1988.

[3] T. Lieuwen and Yang V., (Eds.). Combustion instabilities in gas turbine engines: operational experience, fundamental mechanisms and modeling. Prog. in Astronautics and Aeronautics AIAA, 210, 2005.

[4] A. P. Dowling. The calculation of thermoacoustic oscillations. J. Sound Vib. , 180(4):557-581, 1995.

[5] P. Rao and P. Morris. Use of finite element methods in frequency domain aeroacoustics. AIAA Journal , 44:1643-1652, 2006.

[6] O. Colin, F. Ducros, D. Veynante, and T. Poinsot. A thickened flame model for large eddy simulations of turbulent premixed combustion. Phys. Fluids , 12(7):1843-1863, 2000.

[7] H. Pitsch and L. Duchamp de la Geneste. Large Eddy Simulation of premixed turbulent combustion using a level-set approach. Proc. Combust. Inst. , 29:2001-2008, 2002.

[8] C. D. Pierce and P. Moin. Progress-variable approach for Large Eddy Simulation of non-premixed turbulent combustion. J. Fluid Mech., 504:73-97, 2004.

[9] L. Selle, G. Lartigue, T. Poinsot, R. Koch, K. U. Schildmacher, W. Krebs, B. Prade, P. Kaufmann, and D. Veynante. Compressible Large-Eddy Simulation of turbulent combustion in complex geometry on unstructured meshes. Combust. Flame, 137(4):489-505, 2004. 
[10] S. Roux, G. Lartigue, T. Poinsot, U. Meier, and C. Bérat. Studies of mean and unsteady flow in a swirled combustor using experiments, acoustic analysis and large-eddy simulations. Combust. Flame, 141:40-54, 2005.

[11] H. Pitsch. Large Eddy Simulation of turbulent combustion. Ann. Rev. Fluid Mech., 38:453-482, 2006.

[12] P. Schmitt, T. Poinsot, B. Schuermans, and K. Geigle. Large Eddy Simulation and experimental study of heat transfer, nitric oxide emissions and combustion instability in a swirled turbulent high pressure burner. J. Fluid Mech. , 570:17-46, 17-46.

[13] C. Martin, L. Benoit, Y. Sommerer, F. Nicoud, and T. Poinsot. LES and acoustic analysis of combustion instability in a staged turbulent swirled combustor. AIAA Journal , 44(4):741-750, 2006.

[14] S. R. Stow and A. P. Dowling. Thermoacoustic oscillations in an annular combustor. In ASME paper, volume 2001-GT-0037, New Orleans, Louisiana, 2001.

[15] S. R. Stow and A. P. Dowling. Modelling of circumferential modal coupling due to helmholtz resonators. In ASME paper, volume 2003-GT-38168, Georgia, USA, 2003.

[16] S. Evesque and W. Polifke. Low-order acoustic modelling for annular combustors: Validation and inclusion of modal coupling. In International Gas Turbine and Aeroengine Congress 6 Exposition, ASME Paper, volume GT-2002-30064, 2002.

[17] S. Evesque, W. Polifke, and C. Pankiewitz. Spinning and azimuthally standing acoustic modes in annular combustors. In 9th AIAA/CEAS Aeroacoustics Conference, volume AIAA paper 2003-3182, 2003.

[18] C. F. Silva, F. Nicoud, T. Schuller, D. Durox, and S. Candel. Combining a helmholtz solver with the flame describing function to assess combustion instability in a premixed swirled combustor. Combust. Flame, 160(9):1743-1754, 2013.

[19] J. Gikadi, T. Sattelmayer, and A. Peschiulli. Effects of the mean flow field on the thermo-acoustic stability of aero-engine combustion chambers. In Proceedings of ASME Turbo Expo 2012, number GT2012-69612. ASME, 2012.

[20] L. Selle, L. Benoit, T. Poinsot, F. Nicoud, and W. Krebs. Joint use of compressible Large-Eddy Simulation and Helmholtz solvers for the analysis of rotating modes in an industrial swirled burner. Combust. Flame, 145(1-2):194-205, 2006.

[21] F. Nicoud, L. Benoit, and C. Sensiau. Acoustic modes in combustors with complex impedances and multidimensional active flames. AIAA Journal , 45:426-441, 2007.

[22] H. S. Tsien. The transfer functions of rocket nozzles. J. American Rocket Society , 22(3):139-143, 1952. 
[23] F. E. Marble and S. Candel. Acoustic disturbances from gas nonuniformities convected through a nozzle. J. Sound Vib. , 55:225-243, 1977.

[24] S. R. Stow, A. P. Dowling, and T. P. Hynes. Reflection of circumferential modes in a choked nozzle. J. Fluid Mech., 467:215-239, 2002.

[25] W. H. Moase, M. J. Brear, and C. Manzie. The forced response of choked nozzles and supersonic diffusers. J. Fluid Mech. , 585(1):281-304, 2007.

[26] I. Duran and S. Moreau. Solution of the quasi-one-dimensional linearized euler equations using flow invariants and the magnus expansion. J. Fluid Mech. , 723:190-231, 2013.

[27] N. Lamarque and T. Poinsot. Boundary conditions fo acoustic eigenmode computations in gas turbine combustion chambers. AIAA Journal , 46(9):2282-2292, 2008.

[28] F. Nicoud and K. Wieczorek. About the zero Mach number assumption in the calculation of thermoacoustic instabilities. International Journal of Spray and Combustion Dynamics, 1:67-112, 2009.

[29] S. V. Patankar. Numerical heat transfer and fluid flow. McGraw Hill, New York, 1980.

[30] K. Wieczorek. Numerical study of Mach number effects on combustion instability. PhD thesis, Université Montpellier II, 2010.

[31] M. Leyko, F. Nicoud, and T. Poinsot. Comparison of direct and indirect combustion noise mechanisms in a model combustor. AIAA Journal , 47(11):2709-2716, 2009.

[32] S. Candel. Analytical Studies of Some Acoustic Problems of Jet Engines. PhD thesis, California Institute of Technology, Pasadena, California, 1972.

[33] AVBP. Code: www.cerfacs.fr/cfd/avbp_code.php and www.cerfacs.fr/cfd/cfdpublications.html.

[34] A. Kaufmann, F. Nicoud, and T. Poinsot. Flow forcing techniques for numerical simulation of combustion instabilities. Combust. Flame, 131:371-385, 2002.

[35] T. Poinsot and S. Lele. Boundary conditions for direct simulation of compressible viscous flows. J. Comput. Phys. , 101(1):104-129, 1992.

[36] Gordon C Oates. Aerothermodynamics of aircraft engine components, volume 3. AIAA, 1985.

[37] O. Leonard and O. Adam. A quasi-one-dimensional CFD model for multistage turbomachines. Journal of Thermal Science, 17:7-20, 2008.

[38] P. Wolf, G. Staffelbach, R. Balakrishnan, A. Roux, and T. Poinsot. Azimuthal instabilities in annular combustion chambers. Proc. of the Summer Program, pages 259-269, 2010.

[39] L. Benoit and F. Nicoud. Numerical assessment of thermo-acoustic instabilities in gas turbines. Int. J. Numer. Meth. Fluids, 47:849-855, 2005. 